\title{
Sliding Mode Cooperative Control for Multirobot Systems: A Finite-Time Approach
}

\author{
Masood Ghasemi and Sergey G. Nersesov \\ Department of Mechanical Engineering, Villanova University, Villanova, PA 19085-1681, USA \\ Correspondence should be addressed to Sergey G. Nersesov; sergey.nersesov@villanova.edu
}

Received 26 July 2013; Revised 27 September 2013; Accepted 28 September 2013

Academic Editor: Xudong Zhao

Copyright (c) 2013 M. Ghasemi and S. G. Nersesov. This is an open access article distributed under the Creative Commons Attribution License, which permits unrestricted use, distribution, and reproduction in any medium, provided the original work is properly cited.

\begin{abstract}
Finite-time stability in dynamical systems theory involves systems whose trajectories converge to an equilibrium state in finite time. In this paper, we use the notion of finite-time stability to apply it to the problem of coordinated motion in multiagent systems. We consider a group of agents described by Euler-Lagrange dynamics along with a leader agent with an objective to reach and maintain a desired formation characterized by steady-state distances between the neighboring agents in finite time. We use graph theoretic notions to characterize communication topology in the network determined by the information flow directions and captured by the graph Laplacian matrix. Furthermore, using sliding mode control approach, we design decentralized control inputs for individual agents that use only data from the neighboring agents which directly communicate their state information to the current agent in order to drive the current agent to the desired steady state. We further extend these results to multiagent systems involving underactuated dynamical agents such as mobile wheeled robots. For this case, we show that while the position variables can be coordinated in finite time, the orientation variables converge to the steady states asymptotically. Finally, we validate our results experimentally using a wheeled mobile robot platform.
\end{abstract}

\section{Introduction}

Finite-time stability of a dynamical system involves trajectories that converge to the desired equilibrium state in finite time and remain there for all further times. As a result, such systems do not possess the property of uniqueness of solutions in backward time, and thus their dynamics are nonLipschitz. Sufficient conditions for finite-time stability were rigorously studied in $[1,2]$ using Hölder continuous Lyapunov functions. Finite-time stabilization of second-order systems was considered in [3, 4]. More recently, researchers have considered finite-time stabilization of higher-order systems [5] as well as finite-time stabilization using output feedback [6].

Sufficient conditions for finite-time stability developed in [1] were further extended to finite-time stability and stabilization of sets for nonlinear dynamical systems in [7]. In this paper, we use these results in the context of coordination in multiagent systems. We consider specifically a group of agents characterized by Euler-Lagrange dynamics along with a leader agent with an objective to reach and maintain the desired formation in finite time. Using graphtheoretic notions $[8,9]$, we describe the communication topology in the network of agents characterized by the information share among the agents. Based on the assumption of a weakly connected graph, we develop a directed graph Laplacian matrix whose properties provide the basis for defining the generalized error states. Furthermore, we design decentralized sliding mode controllers for individual vehicles to drive the error states of the entire multiagent system to the origin in finite time. We show that these sliding mode controllers use only information from the neighboring agents that directly communicate their generalized position and velocity information to the current agent.

Recently, finite-time consensus was studied in [10] for single integrator systems with an objective to reach the state of equipartition [11] (or consensus), that is, equal steady states for all agents. In contrast with [10], our approach is not limited by a particular steady state configuration. In fact, the developed framework places no limitation on the motion of 
the leader and the structure of the desired formation and includes, as a special case, all of the above types of coordinated motion such as flocking, cyclic pursuit, and rendezvous. Furthermore, we have extended our results to multiagent systems composed of underactuated dynamical agents.

We use sliding mode control technique [12] to design decentralized controllers for individual agents to reach the desired formation in finite time. Traditionally, sliding mode control is based on defining the sliding surface as a function of the system states and using finite-time stability theory for sets to ensure that all closed-loop systems trajectories reach this surface in finite time [13-17]. The smooth surface is usually designed in such a way that the closed-loop dynamics restricted to the surface is exponentially stable which ensures that trajectories, after reaching the surface, slide along it to the origin exponentially. The main disadvantage of sliding mode control is chattering around the surface during the sliding phase due to the use of discontinuous sign function which, in practice, is normally approximated by a continuous function. In this paper, we briefly review the results of [18] where finite-time coordination in multiagent systems is achieved by designing nonsmooth sliding surfaces to obtain nonLipschitzian closed-loop dynamics restricted to the surface which leads to the convergence of the system trajectories to the origin in finite time. It appears that the sliding mode control designed for such nonsmooth surfaces can be unbounded in a certain region of the state space. In order to overcome this difficulty, we design auxiliary (smooth) sliding surfaces for the initial conditions in this region such that the sliding mode control predicated on these smooth surfaces is bounded. We show that the trajectories starting in this region will inevitably leave it, and hence switching from the sliding mode control designed for smooth surfaces to the one designed for nonsmooth surfaces guarantees convergence of the closed-loop system trajectories to the desired steady states in finite time.

The main focus of this paper is on extending the above framework to multiagent systems composed of underactuated dynamical agents. We use wheeled mobile robot model of an agent to show that while the position variables can be coordinated in finite time, the orientation variables can only be shown to converge to the steady state asymptotically. We demonstrate the efficacy of our approach using an example involving eight planar mobile robots and the leader with given communication topology and the formation structure. In addition, we provide an experimental validation of our results using wheeled mobile robot platform. Overall, the framework reveals fast converging times without excessive control effort.

\section{Mathematical Preliminaries}

In this section, we introduce notation and definitions and present some key results needed for developing the main results of this paper. Let $\mathbb{R}$ denote the set of real numbers, let $\mathbb{R}^{n}$ denote the set of $n \times 1$ column vectors, let $(\cdot)^{T}$ denote transpose, and let $(\cdot)^{-1}$ denote matrix inverse. Furthermore, let $\dot{\mathscr{D}}, \overline{\mathscr{D}}$, and $\partial \mathscr{D}$ denote the interior, the closure, and the boundary of the set $\mathscr{D} \subset \mathbb{R}^{n}$, respectively. Finally, we write $V^{\prime}(x)$ for the Fréchet derivative of $V$ at $x,\|\cdot\|_{\infty}$ for $\ell_{\infty}$ vector/matrix norm, and $\mathbf{e} \in \mathbb{R}^{n}$ for the ones vector of order $n$, that is, $\mathbf{e} \triangleq[1, \ldots, 1]^{\mathrm{T}}$.

Next, consider the nonlinear dynamical system given by

$$
\dot{x}(t)=f(x(t)), \quad x(0)=x_{0}, \quad t \geq 0,
$$

where $x(t) \in \mathscr{D} \subseteq \mathbb{R}^{n}, t \geq 0$, is the system state vector, $\mathscr{D}$ is an open set, $0 \in \mathscr{D}, f(0)=0$, and $f(\cdot)$ is continuous on $\mathscr{D}$. We denote by $s^{x}(t)$ the solution to (1) with the initial condition at $x \in \mathscr{D}$. We assume that (1) possesses unique solutions in forward time for all initial conditions except possibly the origin. Sufficient conditions for forward uniqueness in the absence of Lipschitz continuity can be found in [19], [20, Section 10], [21], and [22, Section 1].

Definition 1 (see [7]). Consider the nonlinear dynamical system (1). Let $\mathscr{D}_{0} \subset \mathscr{D}$ be a positively invariant set with respect to (1). $\mathscr{D}_{0}$ is finite-time stable if there exist an open neighborhood $\mathcal{N} \subseteq \mathscr{D}$ of $\mathscr{D}_{0}$ and a function $T: \mathcal{N} \backslash$ $\mathscr{D}_{0} \rightarrow(0, \infty)$, called the settling-time function, such that the following statements hold.

(i) Finite-Time Convergence. For every $x \in \mathcal{N} \backslash \mathscr{D}_{0}, s^{x}(t)$ is defined on $[0, T(x)), s^{x}(t) \in \mathcal{N} \backslash \mathscr{D}_{0}$ for all $t \in[0, T(x))$, and $\lim _{t \rightarrow T(x)} \operatorname{dist}\left(s(x, t), \mathscr{D}_{0}\right)=0$.

(ii) Lyapunov Stability. For every open neighborhood $\mathcal{O}_{1} \subseteq \mathcal{N}$ of $\mathscr{D}_{0}$, there exists an open neighborhood $\mathscr{O}_{2} \subseteq \mathcal{O}_{1}$ of $\mathscr{D}_{0}$ such that for every $x \in \mathcal{O}_{2} \backslash \mathscr{D}_{0}, s(t, x) \in \mathcal{O}_{1}$ for all $t \in[0, T(x))$.

Finally, $\mathscr{D}_{0}$ is globally finite-time stable if it is finite-time stable with $\mathcal{N}=\mathscr{D}=\mathbb{R}^{n}$.

The next result provides sufficient conditions for finitetime stability of an invariant set.

Theorem 2 (see [7]). Consider the nonlinear dynamical system (1) and let $\mathscr{D}_{0} \subset \mathscr{D}$ be an invariant set with respect to (1). Assume there exists a continuously differentiable function $V: \mathscr{D} \rightarrow \mathbb{R}$ and real numbers $c>0$ and $\alpha \in(0,1)$ such that $V(x)=0, x \in \mathscr{D}_{0}, V(x)>0, x \in \mathscr{D} \backslash \mathscr{D}_{0}$, and

$$
V^{\prime}(x) f(x) \leq-c(V(x))^{\alpha}, \quad x \in \mathscr{D} .
$$

Then, $\mathscr{D}_{0}$ is finite-time stable. Moreover, if $\mathcal{N}$ is as in Definition 1 and $T: \mathcal{N} \rightarrow[0, \infty)$ is the settling-time function, then

$$
T\left(x_{0}\right) \leq \frac{1}{c(1-\alpha)}\left(V\left(x_{0}\right)\right)^{1-\alpha}, \quad x_{0} \in \mathcal{N},
$$

and $T(\cdot)$ is continuous on $\mathcal{N}$. If, in addition, $\mathscr{D}=\mathbb{R}^{n}, V(\cdot)$ is radially unbounded, and (2) holds on $\mathbb{R}^{n}$, then $\mathscr{D}_{0}$ is globally finite-time stable.

Next, we present some basic definitions and results from the graph theory needed in this paper. For more details, see $[8,23,24]$.

A directed graph $G$ of order $n$ is a pair $(\mathscr{V}, \mathscr{E})$, where $\mathscr{V}$ (vertex set) is a set of $n$ elements called vertices (or nodes) and $\mathscr{E}$ (edge set) is a set of ordered pairs of vertices called edges. 
For $u, v \in \mathscr{V}(G)$, the ordered pair $(u, v) \in \mathscr{E}(G)$ denotes an edge from $u$ to $v$. We assume that $u \neq v$ for any $(u, v) \in \mathscr{E}(G)$, that is, the graph has no self-loops. If $(u, v) \in \mathscr{E}(G)$ implies $(v, u) \in \mathscr{E}(G)$ for any $(u, v) \in \mathscr{E}(G)$, then the graph is called undirected graph or simply graph.

The adjacency matrix of $G$ is defined as $A=\left\{a_{i j}\right\}, i, j=$ $1, \ldots, n$, where $a_{i j}, i \neq j$ is equal to one if $\left(u_{j}, u_{i}\right) \in \mathscr{E}(G)$ for $u_{i}, u_{j} \in \mathscr{V}(G)$ and is equal to zero otherwise. Since, $G$ has no self-loops, $a_{i i}=0, i=1, \ldots, n$. The in-degree, respectively out-degree, of a vertex $u$, denoted by $d_{\text {in }}(u)$, respectively $d_{\text {out }}(u)$, is the number of edges $(v, u)$, respectively $(u, v)$, where $(v, u) \in \mathscr{E}(G)$, respectively $(u, v) \in \mathscr{E}(G)$. Moreover, the in-degree matrix of the directed graph $G$, respectively out-degree matrix of the directed graph $G$, denoted by $D_{\text {in }}$, respectively $D_{\text {out }}$, is defined as a diagonal matrix with $d_{\text {in }}(u)$, respectively $d_{\text {out }}(u)$, for all $u \in \mathscr{V}(G)$ as its diagonal elements.

A path on graph $G$ of length $m$ from $u_{0} \in \mathscr{V}(G)$ to $u_{m} \in$ $\mathscr{V}(G)$ is an ordered set of distinct vertices $\left\{u_{0}, \ldots, u_{m}\right\}$ such that, for any pair of consecutive vertices, $\left(u_{i-1}, u_{i}\right) \in \mathscr{E}(G)$ for all $i=1, \ldots, m$. A directed path on a directed graph $G$ of length $m$ from $u_{0} \in \mathscr{V}(G)$ to $u_{m} \in \mathscr{V}(G)$ is an ordered set of distinct vertices $\left\{u_{0}, \ldots, u_{m}\right\}$ such that, for any directed pair of consecutive vertices, $\left(u_{i-1}, u_{i}\right) \in \mathscr{E}(G)$ for all $i=1, \ldots, m$. A graph $G$ is connected if there exists a path between any two of its vertices. A directed graph $G$ is strongly connected if there exists a directed path between any two of its vertices. Also, a directed graph $G$ is weakly connected if it is not strongly connected and its associated undirected graph obtained by removing directions on edges is connected. For any vertex $u_{i} \in \mathscr{V}(G)$, the reachable set $\mathscr{R}_{i}$ is a set containing $u_{i}$ and all vertices $u_{j} \in \mathscr{V}(G)$ such that there is a directed path from $u_{i}$ to $u_{j}$. The reachable set $\mathscr{R} \subset \mathscr{V}(G)$ is called maximal if there exists a vertex $u \in \mathscr{R}$, such that $\mathscr{R}$ is the biggest reachable set in $\mathscr{V}(G)$ containing $u$. A maximal reachable set is called a reach.

The Kirchhoff matrix or directed Laplacian of a directed graph is defined by $L=D_{\text {in }}-A \in \mathbb{R}^{n \times n}$, where $D_{\text {in }}$ is the in-degree matrix and $A$ is the adjacency matrix of the graph. The normalized directed Laplacian denoted by $\mathscr{L} \in \mathbb{R}^{n \times n}$ is obtained by dividing each row of $L$ by the corresponding diagonal element of $D_{\text {in }}$, if it is not zero. If an element of $D_{\text {in }}$ is zero, then the corresponding row of $L$ consists of only zero elements.

Proposition 3. Let $G$ be a graph of order $n$ and let $L$ denote its Laplacian matrix. Then, zero is an eigenvalue of $L$ and the associated eigenvector is $\mathbf{e}=[1, \ldots, 1]^{\mathrm{T}}$.

Proof. Since every row of $L$ sums up to zero, it follows that $\lambda=0$ and $\eta=\mathbf{e}$ is a valid solution to $\left(\lambda I_{n}-L\right) \eta=0$ which proves the result.

Proposition 4 (see [25]). Let $G$ be a directed graph, let $L$ denote its directed Laplacian matrix, and suppose that $G$ has $k$ reaches. Then, the algebraic and geometric multiplicity of the zero eigenvalue of $L$ is equal to $k$.

Corollary 5. Let $G$ be a strongly connected directed graph and let $L$ denote its directed Laplacian matrix. Then, the algebraic and geometric multiplicity of the zero eigenvalue of $L$ is equal to one.

Proof. Since $G$ is strongly connected, it follows that $G$ has only one reach which includes all vertices of $G$. Now, the result is a direct consequence of Proposition 4.

\section{Coordination Control for Euler-Lagrange Systems}

In this section, we briefly review a general framework for coordination control of a set of fully actuated Euler-Lagrange systems in pursuit of a leader [18]. We design specifically decentralized sliding mode controllers for individual agents that guarantee finite-time coordination. Consider a set of $m$ agents whose individual dynamics are given by

$$
\begin{array}{r}
M_{i}\left(q_{i}(t)\right) \ddot{q}_{i}(t)=f_{i}\left(q_{i}(t), \dot{q}_{i}(t)\right)+u_{i}(t)+g_{i}\left(q_{i}(t), \dot{q}_{i}(t)\right), \\
q_{i}(0)=q_{0 i}, \quad \dot{q}_{i}(0)=\dot{q}_{0 i}, \quad t \geq 0, \quad i=1, \ldots, m,
\end{array}
$$

where $q_{i} \in \mathbb{R}^{n}$ is the vector of generalized coordinates of the $i$ th agent, $M_{i}\left(q_{i}\right) \in \mathbb{R}^{n \times n}$ is the positive definite inertia matrix of the $i$ th agent, $u_{i} \in \mathbb{R}^{n}, t \geq 0$, is the control input for the $i$ th agent, $f_{i}\left(q_{i}, \dot{q}_{i}\right) \in \mathbb{R}^{n}$ is the vector of Coriolis, centrifugal, conservative, and nonconservative forces acting on the $i$ th agent, and $g_{i}\left(q_{i}, \dot{q}_{i}\right) \in \mathbb{R}^{n}$ represents the vector of bounded uncertainties and disturbances affecting the dynamics of the $i$ th agent. Alternatively, (4) can be written as

$$
\begin{gathered}
\ddot{q}_{i}(t)=\tilde{f}_{i}\left(q_{i}(t), \dot{q}_{i}(t)\right)+\tilde{u}_{i}(t)+\tilde{g}_{i}\left(q_{i}(t), \dot{q}_{i}(t)\right), \\
q_{i}(0)=q_{0 i}, \quad \dot{q}_{i}(0)=\dot{q}_{0 i}, \quad t \geq 0, \quad i=1, \ldots, m,
\end{gathered}
$$

where

$$
\begin{gathered}
\tilde{f}_{i}\left(q_{i}, \dot{q}_{i}\right) \triangleq M_{i}^{-1}\left(q_{i}\right) f_{i}\left(q_{i}, \dot{q}_{i}\right), \\
\tilde{u}_{i} \triangleq M_{i}^{-1}\left(q_{i}\right) u_{i}, \\
\widetilde{g}_{i}\left(q_{i}, \dot{q}_{i}\right) \triangleq M_{i}^{-1}\left(q_{i}\right) g_{i}\left(q_{i}, \dot{q}_{i}\right) .
\end{gathered}
$$

We associate with the network of agents, including the leader agent, the directed graph $G$ and use subscripts $1, \ldots, m$ to refer to the agents and "L" to refer to the leader agent. Next, define the set $\mathscr{F}_{i}$ given by

$$
\mathscr{F}_{i} \subset\{\mathrm{L}, 1, \ldots, m\} \backslash\{i\}, \quad i=1, \ldots, m,
$$

to denote the set of all agents whose position and velocity vectors are available for the $i$ th agent, that is, $(j, i) \in \mathscr{E}(G)$ for all $j \in \mathscr{J}_{i}$. We assume that the directed graph $G$ of the ensemble of agents is such that there exists at least one directed path from the leader to any agent, which implies that $G$ is weakly connected. Note that the leader, where the information flow starts, does not sense any agent, that is, there is no directed path from any agent to the leader. Moreover, we assume that the communication links between all agents remain unchanged. Thus, the graph topology of the agent 
network is static. The normalized directed Laplacian matrix $\mathscr{L} \in \mathbb{R}^{(m+1) \times(m+1)}$ of the graph is given by

$$
\mathscr{L}_{i j}= \begin{cases}1, & i=j, i \neq \mathrm{L}, \\ -\frac{1}{\left|\mathscr{J}_{i}\right|}, & j \in \mathscr{J}_{i}, i \neq \mathrm{L}, \\ 0, & \text { otherwise, }\end{cases}
$$

where $\left|\mathscr{F}_{i}\right|$ is the cardinality of $\mathscr{F}_{i}, i=1, \ldots, m$, which is the number of elements in $\mathscr{F}_{i}$. Note that, since the leader does not sense any agent, the first row of the Laplacian matrix is zero. Alternatively, the Laplacian matrix (8) can be rewritten as

$$
\mathscr{L}=\left[\begin{array}{cc}
0 & 0_{1 \times m} \\
L_{1} & L_{2}
\end{array}\right],
$$

where

$$
\begin{gathered}
L_{1}=\left[\mathscr{L}_{1 \mathrm{~L}}, \ldots, \mathscr{L}_{m \mathrm{~L}}\right]^{\mathrm{T}}, \\
L_{2}=\left[\begin{array}{ccc}
\mathscr{L}_{11} & \cdots & \mathscr{L}_{1 m} \\
\vdots & \ddots & \vdots \\
\mathscr{L}_{m 1} & \cdots & \mathscr{L}_{m m}
\end{array}\right],
\end{gathered}
$$

and $\mathscr{L}_{i j}, i=1, \ldots, m, j=\mathrm{L}, 1, \ldots, m$, are given by (8). The next lemma presents the key feature of the matrix $L_{2} \in \mathbb{R}^{m \times m}$.

Lemma 6 (see [18]). Let the connectivity graph $G$ have the normalized directed Laplacian matrix $\mathscr{L} \in \mathbb{R}^{(m+1) \times(m+1)}$ given by (9). Then, $L_{2} \in \mathbb{R}^{m \times m}$ is invertible.

We consider an objective where the agents whose dynamics are given by (4) are required to achieve and maintain coordinated motion with respect to the leader in finite time. We consider specifically a formation leader whose position and velocity profiles are known functions of time and are given by $q_{\mathrm{L}}(t), t \geq 0$, and $\dot{q}_{\mathrm{L}}(t), t \geq 0$. In this case, the desired steady state value of the $i$ th agent relative position with respect to the $j$ th agent is uniquely defined by a known vector $l_{i j}(t) \in \mathbb{R}^{n}, t \geq 0, i, j=\mathrm{L}, 1, \ldots, m$, characterizing the time varying difference between the $i$ th and the $j$ th agents' generalized position vectors. Thus, the error variable that needs to be driven to zero is given by

$$
e_{i j}(t) \triangleq q_{i}(t)-q_{j}(t)-l_{i j}(t), \quad i, j=\mathrm{L}, 1, \ldots, m, t \geq 0,
$$

where $e_{i j}(t) \in \mathbb{R}^{n}, t \geq 0$ is the position error of the $i$ th agent with respect to the $j$ th agent. Note that, at this point, $e_{i j}, i, j=$ $L, 1, \ldots, m$, are defined regardless of whether $j \in \mathscr{F}_{i}$ or not. Furthermore, we introduce a generalized error state for the $i$ th agent as

$$
z_{i}(t) \triangleq q_{i}(t)-\frac{1}{\left|\mathscr{J}_{i}\right|} \sum_{j \in \mathscr{F}_{i}}\left(q_{j}(t)+l_{i j}(t)\right), \quad i=1, \ldots, m .
$$

Note that in contrast with (11), in (12), the generalized position $q_{i}, i=1, \ldots, m$, is compared to only the generalized positions $q_{j}$, where $j \in \mathscr{J}_{i}$.
Lemma 7 (see [18]). Consider the connectivity graph $G$ with the normalized directed Laplacian matrix $\mathscr{L} \in \mathbb{R}^{(m+1) \times(m+1)}$ given by (9). Define $z \triangleq\left[z_{1}^{\mathrm{T}}, \ldots, z_{m}^{\mathrm{T}}\right]^{\mathrm{T}}$, where $z_{i} \in \mathbb{R}^{n}$ is given by (12) and $e_{j} \triangleq\left[e_{1 j}^{\mathrm{T}}, \ldots, e_{m j}^{\mathrm{T}}\right]^{\mathrm{T}}, j=\mathrm{L}, 1, \ldots, m$. Then, the following statements hold.

(i) If $z=0$, then $e_{i j}=0, i=1, \ldots, m, j=\mathrm{L}, 1, \ldots, m$.

(ii) If $e_{i j}=0, i=1, \ldots, m, j \in \mathscr{F}_{i}$, then $z=0$.

The generalized error dynamics are obtained by taking the second time derivative of (12) and are given by

$$
\begin{gathered}
\ddot{z}_{i}(t)=v_{i}(t)-\ddot{l}_{i}(t)+\tilde{f}_{i}\left(z, \dot{z}, q_{\mathrm{L}}(t), \dot{q}_{\mathrm{L}}(t)\right) \\
-\frac{1}{\left|\mathscr{g}_{i}\right|} \sum_{j \in \mathscr{f}_{i} \mid\{\mathrm{L}\}} \tilde{f}_{j}\left(z, \dot{z}, q_{\mathrm{L}}(t), \dot{q}_{\mathrm{L}}(t)\right)+\mathscr{L}_{i \mathrm{~L}} \ddot{q}_{\mathrm{L}}(t) \\
+h_{i}\left(z, \dot{z}, q_{\mathrm{L}}(t), \dot{q}_{\mathrm{L}}(t)\right), \\
z_{i}(0)=z_{0 i}, \quad \dot{z}_{i}(0)=\dot{z}_{0 i}, \quad i=1, \ldots, m, t \geq 0,
\end{gathered}
$$

where

$$
\begin{array}{r}
v_{i}(t) \triangleq \widetilde{u}_{i}(t)-\frac{1}{\left|\mathscr{F}_{i}\right|} \sum_{j \in \mathscr{F}_{i} \backslash\{\mathrm{L}\}} \widetilde{u}_{j}(t), \quad i=1, \ldots, m, \\
l_{i}(t) \triangleq \frac{1}{\left|\mathscr{F}_{i}\right|} \sum_{j \in \mathscr{F}_{i}} l_{i j}(t), \quad i=1, \ldots, m, \\
h_{i}\left(z, \dot{z}, q_{\mathrm{L}}(t), \dot{q}_{\mathrm{L}}(t)\right) \\
\triangleq \widetilde{g}_{i}\left(q_{i}(t), \dot{q}_{i}(t)\right)-\frac{1}{\left|\mathscr{F}_{i}\right|} \sum_{j \in \mathscr{F}_{i} \backslash\{\mathrm{L}\}} \widetilde{g}_{j}\left(q_{j}(t), \dot{q}_{j}(t)\right), \\
\quad i=1, \ldots, m .
\end{array}
$$

Equations (12), (13), and (14) for individual agents can be rewritten using Kronecker algebra to represent the multiagent formation consisting of $m$ agents as follows:

$$
\begin{gathered}
z(t) v=\left(L_{2} \otimes I_{n}\right) q(t)+L_{1} \otimes q_{\mathrm{L}}(t)-l(t), \\
\ddot{z}(t)=v(t)-\ddot{l}(t)+\left(L_{2} \otimes I_{n}\right) F\left(z, \dot{z}, q_{\mathrm{L}}(t), \dot{q}_{\mathrm{L}}(t)\right) \\
+L_{1} \otimes \ddot{q}_{\mathrm{L}}(t)+h\left(z, \dot{z}, q_{\mathrm{L}}(t), \dot{q}_{\mathrm{L}}(t)\right), \\
z(0)=z_{0}, \quad \dot{z}(0)=\dot{z}_{0}, \quad t \geq 0, \\
v(t)=\left(L_{2} \otimes I_{n}\right) \tilde{u}(t),
\end{gathered}
$$


where

$$
\begin{gathered}
q(t) \triangleq\left[q_{1}^{\mathrm{T}}(t), \ldots, q_{m}^{\mathrm{T}}(t)\right]^{\mathrm{T}}, \\
l(t) \triangleq\left[l_{1}^{\mathrm{T}}(t), \ldots, l_{m}^{\mathrm{T}}(t)\right]^{\mathrm{T}}, \\
v(t) \triangleq\left[v_{1}^{\mathrm{T}}(t), \ldots, v_{m}^{\mathrm{T}}(t)\right]^{\mathrm{T}}, \\
\widetilde{u}(t) \triangleq\left[\tilde{u}_{1}^{\mathrm{T}}(t), \ldots, \widetilde{u}_{m}^{\mathrm{T}}(t)\right]^{\mathrm{T}}, \\
F\left(z, \dot{z}, q_{\mathrm{L}}(t), \dot{q}_{\mathrm{L}}(t)\right) \\
\triangleq\left[\tilde{f}_{1}^{\mathrm{T}}\left(z, \dot{z}, q_{\mathrm{L}}(t), \dot{q}_{\mathrm{L}}(t)\right), \ldots, \widetilde{f}_{m}^{\mathrm{T}}\left(z, \dot{z}, q_{\mathrm{L}}(t), \dot{q}_{\mathrm{L}}(t)\right)\right]^{\mathrm{T}}, \\
h\left(z, \dot{z}, q_{\mathrm{L}}(t), \dot{q}_{\mathrm{L}}(t)\right) \\
\triangleq\left[h_{1}^{\mathrm{T}}\left(z, \dot{z}, q_{\mathrm{L}}(t), \dot{q}_{\mathrm{L}}(t)\right), \ldots, h_{m}^{\mathrm{T}}\left(z, \dot{z}, q_{\mathrm{L}}(t), \dot{q}_{\mathrm{L}}(t)\right)\right]^{\mathrm{T}} .
\end{gathered}
$$

Next, we present a general framework for the sliding mode control design that guarantees finite-time coordination for the multiagent system (17). Consider a vector function $\sigma_{i}: \mathbb{R}^{n} \times \mathbb{R}^{n} \rightarrow \mathbb{R}^{n}$ given by

$$
\sigma_{i}\left(z_{i}, \dot{z}_{i}\right)=\dot{z}_{i}+C_{i} S_{i}\left(z_{i}\right)\left|z_{i}\right|^{1 / 2}, \quad\left(z_{i}, \dot{z}_{i}\right) \in \mathbb{R}^{n} \times \mathbb{R}^{n},
$$

where $C_{i}=\operatorname{diag}\left[c_{i 1}, \ldots, c_{i n}\right], c_{i j}>0, i=1, \ldots, m, j=$ $1, \ldots, n, S_{i}\left(z_{i}\right) \triangleq \operatorname{diag}\left[\operatorname{sign}\left(z_{i 1}\right), \ldots, \operatorname{sign}\left(z_{i n}\right)\right], z_{i j} \in \mathbb{R}, i=$ $1, \ldots, m, j=1, \ldots, n$, is the $j$ th component of $z_{i} \in \mathbb{R}^{n}$, $\left|z_{i}\right|^{1 / 2} \triangleq\left[\left|z_{i 1}\right|^{1 / 2}, \ldots,\left|z_{i n}\right|^{1 / 2}\right]^{\mathrm{T}}$. We define the $i$ th sliding surface as the null space of $\sigma_{i}(\cdot, \cdot)$, that is,

$$
\mathcal{S}_{i} \triangleq\left\{\left(z_{i}, \dot{z}_{i}\right) \in \mathbb{R}^{n} \times \mathbb{R}^{n}: \sigma_{i}\left(z_{i}, \dot{z}_{i}\right)=0\right\} .
$$

As in standard sliding mode control theory, the control law $v_{i}$ is calculated by setting $\dot{\sigma}_{i}\left(z_{i}, \dot{z}_{i}\right)=0$ for the nominal system and adding a signum function to address uncertainties. Using (13) and (20) specifically, we set

$$
\begin{aligned}
v_{i}(t)= & \ddot{l}_{i}(t)-\tilde{f}_{i}\left(z, \dot{z}, q_{\mathrm{L}}(t), \dot{q}_{\mathrm{L}}(t)\right) \\
& +\frac{1}{\left|\mathscr{f}_{i}\right|} \sum_{j \in \mathscr{f}_{i} \backslash\{\mathrm{L}\}} \tilde{f}_{j}\left(z, \dot{z}, q_{\mathrm{L}}(t), \dot{q}_{\mathrm{L}}(t)\right)-\mathscr{L}_{i \mathrm{~L}} \ddot{q}_{\mathrm{L}}(t) \\
& -\frac{1}{2} C_{i} d_{i}\left(z_{i}, \dot{z}_{i}\right)-K_{i} \operatorname{sign}\left(\sigma_{i}\left(z_{i}, \dot{z}_{i}\right)\right), \\
& \left(z_{i}, \dot{z}_{i}\right) \in \mathscr{M}_{i}, i=1, \ldots, m, \quad\left(z_{j}, \dot{z}_{j}\right) \in \mathbb{R}^{n} \times \mathbb{R}^{n}, \\
& j=1, \ldots, m, j \neq i,
\end{aligned}
$$

where $K_{i}=\left[k_{i 1}, \ldots, k_{i n}\right], k_{i j} \in \mathbb{R}, i=1, \ldots, m, j=1, \ldots, n$,

$$
\begin{aligned}
& \operatorname{sign}\left(\sigma_{i}\left(z_{i}, \dot{z}_{i}\right)\right) \triangleq\left[\begin{array}{c}
\operatorname{sign}\left(\sigma_{i 1}\left(z_{i}, \dot{z}_{i}\right)\right) \\
\vdots \\
\operatorname{sign}\left(\sigma_{\text {in }}\left(z_{i}, \dot{z}_{i}\right)\right)
\end{array}\right], \\
& d_{i}\left(z_{i}, \dot{z}_{i}\right) \triangleq\left[\begin{array}{c}
\dot{z}_{i 1}\left|z_{i 1}\right|^{-1 / 2} \\
\vdots \\
\dot{z}_{\text {in }}\left|z_{\text {in }}\right|^{-1 / 2}
\end{array}\right],
\end{aligned}
$$

$\sigma_{i j}(\cdot, \cdot), i=1, \ldots, m, j=1, \ldots, n$, is the $j$ th component of $\sigma_{i}(\cdot, \cdot)$, and $\mathscr{M}_{i} \subset \mathbb{R}^{n} \times \mathbb{R}^{n}$ is the set where $d_{i}(\cdot, \cdot)$ is bounded. Define $\mathscr{M}_{i}, i=1, \ldots, m$, as

$$
\mathscr{M}_{i} \triangleq\left\{\left(z_{i}, \dot{z}_{i}\right) \in \mathbb{R}^{n} \times \mathbb{R}^{n}:\left\|d_{i}\left(z_{i}, \dot{z}_{i}\right)\right\|_{\infty} \leq \lambda_{i}\right\},
$$

where

$$
\lambda_{i} \triangleq\left\|C_{i}\right\|_{\infty}+\delta_{i}, \quad \delta_{i}>0
$$

Note that vector $d_{i}(\cdot, \cdot)$ and, consequently, $v_{i}$ are bounded in $\mathscr{M}_{i} \subset \mathbb{R}^{n} \times \mathbb{R}^{n}$ while being unbounded in the complement of $\mathscr{M}_{i}$. Thus, for the complement of $\mathscr{M}_{i}$, we design an auxiliary sliding mode controller below. First, however, we establish sufficient conditions under which $\mathscr{M}_{i}$ is positively invariant with respect to (13).

Proposition 8 (see [18]). Consider the error dynamics (13) with the feedback controller (22). If the sliding mode controller gains $k_{i j}, i=1, \ldots, m, j=1, \ldots, n$, satisfy

$$
k_{i j}=\eta_{i j}+\sup _{(z, \dot{z}, t) \in \mathbb{R}^{n m} \times \mathbb{R}^{n m} \times \mathbb{R}}\left\|h_{i}\left(z, \dot{z}, q_{\mathrm{L}}(t), \dot{q}_{\mathrm{L}}(t)\right)\right\|_{\infty}
$$

with

$$
\eta_{i j}>\frac{\lambda_{i}^{2}-c_{i j} \lambda_{i}}{2}>0
$$

then the set $\mathscr{M}_{i}$ defined by (24) is invariant with respect to (13).

For the case when $\left(z_{i}, \dot{z}_{i}\right) \in \mathscr{M}_{i}, i=1, \ldots, m$, decentralized control input (22) can be rewritten for the entire ensemble of $m$ agents as

$$
\begin{gathered}
v(t)=\ddot{l}(t)-\frac{1}{2} C d(z, \dot{z})+\left(L_{2} \otimes I_{n}\right) F\left(z, \dot{z}, q_{\mathrm{L}}(t), \dot{q}_{\mathrm{L}}(t)\right) \\
-L_{1} \otimes \ddot{q}_{\mathrm{L}}(t)-K \operatorname{sign}(\sigma(z, \dot{z})), \\
\left(z_{i}, \dot{z}_{i}\right) \in \mathscr{M}_{i}, \quad i=1, \ldots, m,
\end{gathered}
$$


where

$$
\begin{aligned}
& K \triangleq \text { block }-\operatorname{diag}\left[K_{1}, \ldots, K_{m}\right], \\
& C \triangleq \operatorname{block}-\operatorname{diag}\left[C_{1}, \ldots, C_{m}\right], \\
& d(z, \dot{z}) \triangleq\left[d_{1}^{\mathrm{T}}\left(z_{1}, \dot{z}_{1}\right), \ldots, d_{m}^{\mathrm{T}}\left(z_{m}, \dot{z}_{m}\right)\right]^{\mathrm{T}}, \\
& \sigma(z, \dot{z}) \triangleq\left[\sigma_{1}^{\mathrm{T}}\left(z_{1}, \dot{z}_{1}\right), \ldots, \sigma_{m}^{\mathrm{T}}\left(z_{m}, \dot{z}_{m}\right)\right]^{\mathrm{T}},
\end{aligned}
$$

$\operatorname{sign}(\sigma(z, \dot{z}))$

$$
\triangleq\left[\left(\operatorname{sign}\left(\sigma_{1}\left(z_{1}, \dot{z}_{1}\right)\right)\right)^{\mathrm{T}}, \ldots,\left(\operatorname{sign}\left(\sigma_{m}\left(z_{m}, \dot{z}_{m}\right)\right)\right)^{\mathrm{T}}\right]^{\mathrm{T}} .
$$

Next, for the initial conditions $\left(z_{0 i}, \dot{z}_{0 i}\right) \in \mathbb{R}^{n} \times \mathbb{R}^{n} \backslash \mathscr{M}_{i}$, we design an auxiliary sliding surface $\mathcal{S}_{\text {iaux }} \subset \mathbb{R}^{n} \times \mathbb{R}^{n}$ along with the corresponding sliding mode controller. Consider specifically the vector function $\sigma_{\text {iaux }}: \mathbb{R}^{n} \times \mathbb{R}^{n} \rightarrow \mathbb{R}^{n}$ given by

$$
\sigma_{\text {iaux }}\left(z_{i}, \dot{z}_{i}\right)=\dot{z}_{i}, \quad\left(z_{i}, \dot{z}_{i}\right) \in \mathbb{R}^{n} \times \mathbb{R}^{n}
$$

and define the sliding surface $\delta_{\text {iaux }}$ as

$$
\mathcal{S}_{\text {iaux }} \triangleq\left\{\left(z_{i}, \dot{z}_{i}\right) \in \mathbb{R}^{n} \times \mathbb{R}^{n}: \sigma_{\text {iaux }}\left(z_{i}, \dot{z}_{i}\right)=0\right\}
$$

By setting $\dot{\sigma}_{\text {iaux }}\left(z_{i}, \dot{z}_{i}\right)=0$, we obtain

$$
\begin{aligned}
v_{\text {iaux }}= & \ddot{l}_{i}(t)-\tilde{f}_{i}\left(z, \dot{z}, q_{\mathrm{L}}(t), \dot{q}_{\mathrm{L}}(t)\right) \\
& +\frac{1}{\left|\mathscr{J}_{i}\right|} \sum_{j \in \mathcal{F}_{i} \backslash\{\mathrm{L}\}} \tilde{f}_{j}\left(z, \dot{z}, q_{\mathrm{L}}(t), \dot{q}_{\mathrm{L}}(t)\right) \\
& -\mathscr{L}_{i \mathrm{~L}} \ddot{q}_{\mathrm{L}}(t)-K_{i \mathrm{aux}} \operatorname{sign}\left(\sigma_{i \mathrm{aux}}\left(z_{i}, \dot{z}_{i}\right)\right), \\
& \left(z_{i}, \dot{z}_{i}\right) \notin \mathscr{M}_{i}, \quad i=1, \ldots, m, \\
& \left(z_{j}, \dot{z}_{j}\right) \in \mathbb{R}^{n} \times \mathbb{R}^{n}, \quad j=1, \ldots, m, j \neq i
\end{aligned}
$$

where $K_{\text {iaux }} \triangleq \operatorname{diag}\left[k_{i 1 \text { aux }}, \ldots, k_{\text {in aux }}\right], i=1, \ldots, m$,

$$
\begin{array}{r}
k_{i j a u x}=\eta_{i j a u x}+\sup _{\left(z_{i}, \dot{z}_{i}, t\right) \in \mathbb{R}^{n} \times \mathbb{R}^{n} \times \mathbb{R}}\left\|h\left(z, \dot{z}, q_{\mathrm{L}}(t), \dot{q}_{\mathrm{L}}(t)\right)\right\|_{\infty}, \\
j=1, \ldots, n,
\end{array}
$$

with $\eta_{i j a u x}>0$. The next result establishes that the sliding mode controllers (22) and (32) guarantee that the error states $(z, \dot{z})$ of (17) converge to the origin in finite time, thus ensuring finite-time coordination among the agents.
Theorem 9 (see [18]). Consider the error dynamics given by (13). The sliding mode control law given by

$v_{i}(t)$

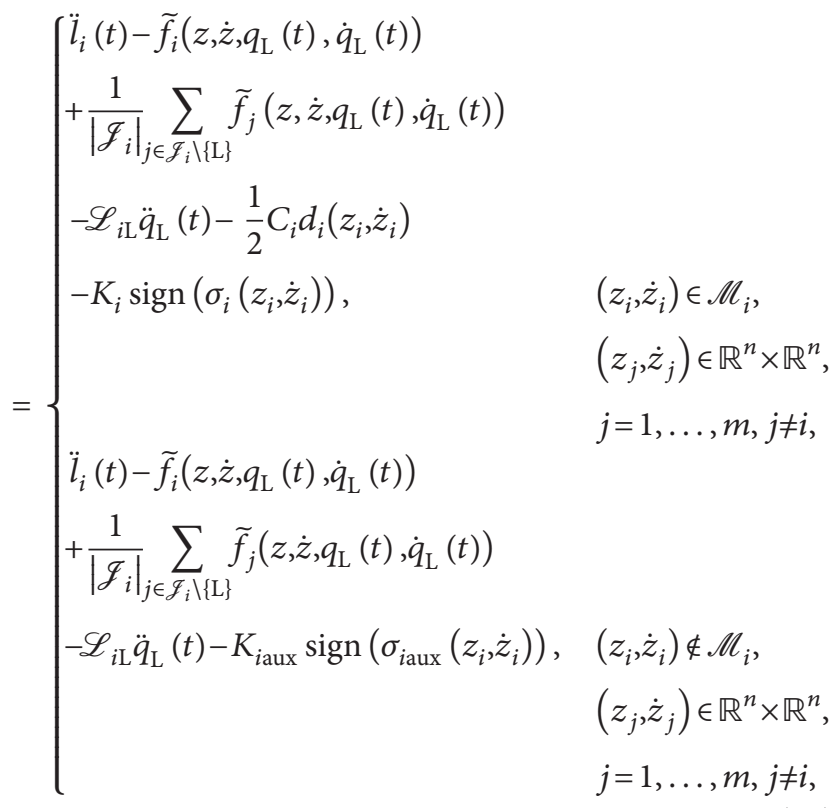

where $i=1, \ldots, m, \sigma_{i}(\cdot, \cdot)$ and $\sigma_{i \text { aux }}(\cdot, \cdot)$ are given by $(20)$ and (30), respectively, the entries of control gain matrices $K_{i} \epsilon$ $\mathbb{R}^{n \times n}$ satisfy (26) and (27), and the entries of control gain matrices $K_{\text {iaux }} \in \mathbb{R}^{n \times n}$ satisfy (33), guarantees finite-time convergence of the error state $(z, \dot{z})$ of $(17)$ to the origin, and hence, the ensemble of $m$ agents and a leader reaches the desired coordination in finite time.

Note that each decentralized controller $v_{i}, i=1, \ldots, m$, given by (34) uses only local information from the $i$ th agent and the neighboring agents that directly communicate their state information to the $i$ th agent. Furthermore, the earlier statement that the leader does not receive information from the agents is not restrictive. In fact, if the agents do communicate their state values to the leader, then as long as the leader's position and velocity are known by at least one agent, the analysis will be the same as presented above.

Remark 10. Sliding mode control always results in chattering around the surfaces due to the signum function in (34). In practice, the discontinuous sign function is often approximated by a continuous function such as the hyperbolic tangent or by a linear saturation function to avoid chattering.

\section{Finite-Time Coordination for Underactuated Systems}

In this section, we extend the results of Section 3 to the case of underactuated mechanical systems. As an example system, we consider a model of a mobile robot subject to a nonholonomic constraint such as the no-slip condition in the 
normal direction to the motion path. The equations of motion for a mobile robot shown in Figure 1 are given by [26]

$$
\begin{gathered}
\dot{x}_{i}(t)=v_{x i}(t) \cos \theta_{i}(t)-d \omega_{i}(t) \sin \theta_{i}(t), \\
x_{i}(0)=x_{0 i}, \quad i=1, \ldots, p, t \geq 0, \\
\dot{y}_{i}(t)=v_{x i}(t) \sin \theta_{i}(t)+d \omega_{i}(t) \cos \theta_{i}(t), \\
y_{i}(0)=y_{0 i}, \\
\dot{\theta}_{i}(t)=\omega_{i}(t), \quad \theta_{i}(0)=\theta_{0 i}, \\
\dot{v}_{x i}(t)=\frac{m d}{\widetilde{m}} \omega_{i}^{2}(t)+\frac{1}{\widetilde{m} r}\left(\tau_{1 i}(t)+\tau_{2 i}(t)\right) \\
+g_{1 i}\left(\eta_{1 i}, \eta_{2 i}\right), \quad v_{x i}(0)=v_{0 x i}, \\
\dot{\omega}_{i}(t)=-\frac{m d}{\widetilde{I}} \omega_{i}(t) v_{x i}(t)+\frac{a}{2 \widetilde{I} r}\left(\tau_{2 i}(t)-\tau_{1 i}(t)\right) \\
+g_{2 i}\left(\eta_{1 i}, \eta_{2 i}\right), \quad \omega(0)=\omega_{0},
\end{gathered}
$$

where $\left(x_{i}, y_{i}\right)$ is the position of the $i$ th robot's mass center $C, \theta_{i}$ is the $i$ th robot's orientation (see Figure 1), $v_{x i}$ is the tangential component of the velocity of the robot's mass center, $\tau_{1 i}$ and $\tau_{2 i}$ are differential torques applied to each wheel, $g_{1 i}(\cdot, \cdot)$ and $g_{2 i}(\cdot, \cdot)$ are uncertainty functions affecting the robot dynamics, $\eta_{1 i} \triangleq\left[x_{i}, y_{i}, \theta_{i}\right]^{\mathrm{T}}, \eta_{2 i} \triangleq\left[v_{x i}, \omega_{i}\right]^{\mathrm{T}}, d>0$ is the distance between the mass center $C$ and mid-axle point $A, m$ is the total mass of the robot, $I$ is the moment of inertia of the robot about the axis orthogonal to the plane passing through $C, a$ is the length of robot's axle, $\widetilde{m} \triangleq m+2 J / r^{2}$ and $\widetilde{I} \triangleq I+m d^{2}+a^{2} / r^{2} J, J$ is the rotational inertia of the wheels about their axis of rotation, $r$ is the radius of the wheels, and $p$ is the number of mobile robots. Differentiating (35) and (36) yields

$$
\begin{gathered}
\ddot{x}_{i}(t)=f_{1 i}\left(\eta_{1 i}(t), \eta_{2 i}(t)\right)+u_{1 i}(t) \cos \theta_{i}(t) \\
-d u_{2 i}(t) \sin \theta_{i}(t)+\widetilde{g}_{1 i}\left(\eta_{1 i}(t), \eta_{2 i}(t)\right), \\
x_{i}(0)=x_{0 i}, \quad \dot{x}_{i}(0)=\dot{x}_{0 i}, \\
i=1, \ldots, p, t \geq 0, \\
\ddot{y}_{i}(t)=f_{2 i}\left(\eta_{1 i}(t), \eta_{2 i}(t)\right)+u_{1 i}(t) \sin \theta_{i}(t) \\
+d u_{2 i}(t) \cos \theta_{i}(t)+\widetilde{g}_{2 i}\left(\eta_{1 i}(t), \eta_{2 i}(t)\right), \\
y_{i}(0)=y_{0 i}, \quad \dot{y}_{i}(0)=\dot{y}_{0 i},
\end{gathered}
$$

where

$$
\begin{aligned}
u_{1 i} \triangleq & \frac{1}{\widetilde{m} r}\left(\tau_{1 i}+\tau_{2 i}\right), \quad i=1, \ldots, p, \\
u_{2 i} \triangleq & \frac{a}{2 \widetilde{I} r}\left(\tau_{2 i}-\tau_{1 i}\right), \quad i=1, \ldots, p, \\
f_{1 i}\left(\eta_{1 i}, \eta_{2 i}\right) \triangleq & \left(\frac{m}{\widetilde{m}}-1\right) d \omega_{i}^{2} \cos \theta_{i} \\
& +\left(\frac{m d^{2}}{\widetilde{I}}-1\right) \omega_{i} v_{x i} \sin \theta_{i}, \quad i=1, \ldots, p,
\end{aligned}
$$

$$
\begin{aligned}
f_{2 i}\left(\eta_{1 i}, \eta_{2 i}\right) \triangleq & \left(\frac{m}{\widetilde{m}}-1\right) d \omega_{i}^{2} \sin \theta_{i} \\
& -\left(\frac{m d^{2}}{\widetilde{I}}-1\right) \omega_{i} v_{x i} \cos \theta_{i}, \quad i=1, \ldots, p, \\
\widetilde{g}_{1 i}\left(\eta_{1 i}, \eta_{2 i}\right) \triangleq & g_{1 i}\left(\eta_{1 i}, \eta_{2 i}\right) \cos \theta_{i} \\
& -d g_{2 i}\left(\eta_{1 i}, \eta_{2 i}\right) \sin \theta_{i}, \quad i=1, \ldots, p, \\
\widetilde{g}_{2 i}\left(\eta_{1 i}, \eta_{2 i}\right) \triangleq & g_{1 i}\left(\eta_{1 i}, \eta_{2 i}\right) \sin \theta_{i} \\
& +d g_{2 i}\left(\eta_{1 i}, \eta_{2 i}\right) \cos \theta_{i}, \quad i=1, \ldots, p .
\end{aligned}
$$

Next, using the feedback linearizing controller given by

$$
\begin{array}{r}
{\left[\begin{array}{l}
u_{1 i} \\
u_{2 i}
\end{array}\right]=\left[\begin{array}{cc}
\cos \theta_{i} & -d \sin \theta_{i} \\
\sin \theta_{i} & d \cos \theta_{i}
\end{array}\right]^{-1}\left[\begin{array}{l}
-f_{1 i}\left(\eta_{1 i}, \eta_{2 i}\right)+v_{1 i} \\
-f_{2 i}\left(\eta_{1 i}, \eta_{2 i}\right)+v_{2 i}
\end{array}\right],} \\
i=1, \ldots, p,
\end{array}
$$

where $v_{1 i} \in \mathbb{R} i=1, \ldots, p$, and $v_{2 i} \in \mathbb{R} i=1, \ldots, p$, are the new feedback control inputs, (40) can be written as

$$
\begin{gathered}
\ddot{x}_{i}(t)=v_{1 i}(t)+\widetilde{g}_{1 i}\left(\eta_{1 i}(t), \eta_{2 i}(t)\right), \\
x_{i}(0)=x_{0 i}, \quad \dot{x}_{i}(0)=\dot{x}_{0 i}, \quad i=1, \ldots, p, \\
\ddot{y}_{i}(t)=v_{2 i}(t)+\widetilde{g}_{2 i}\left(\eta_{1 i}(t), \eta_{2 i}(t)\right), \\
y_{i}(0)=y_{0 i}, \quad \dot{y}_{i}(0)=\dot{y}_{0 i},
\end{gathered}
$$

while (37) can be rewritten as

$$
\begin{array}{r}
\dot{\theta}_{i}(t)=-\frac{1}{d}\left(\dot{x}_{i}(t) \sin \theta_{i}(t)-\dot{y}_{i}(t) \cos \theta_{i}(t)\right), \\
\theta_{i}(0)=\theta_{0 i}, \quad t \geq 0, \quad i=1, \ldots, p .
\end{array}
$$

The objective is to coordinate the motion of a set of $p$ robots and a leader. Define the leader's position in the plane as $\left(x_{\mathrm{L}}(t), y_{\mathrm{L}}(t)\right), t \geq 0$, and its orientation as $\theta_{\mathrm{L}}(t), t \geq 0$, and define the generalized position vector for the leader as $q_{\mathrm{L}}(t)=\left[x_{\mathrm{L}}(t), y_{\mathrm{L}}(t), \theta_{\mathrm{L}}(t)\right]^{\mathrm{T}}, t \geq 0$. Note that the leader obeys the same nonholonomic constraint as all mobile robots such as no slip in the normal direction to the path. Without loss of generality, we assume that $v_{x \mathrm{~L}}(t)>0, t \geq 0$, which physically implies that, during the motion of the leader, its mass center $C$ is always ahead of the mid-axle point $A$. In other words, in order for the leader robot to turn around, it does not back up the wheels, but uses the differential torques applied to the wheels while keeping $v_{x \mathrm{~L}}>0$. As in Section 3, we define the set $\mathscr{F}_{i}$ given by

$$
\mathscr{F}_{i} \subset\{\mathrm{L}, 1, \ldots, p\} \backslash\{i\}, \quad i=1, \ldots, p,
$$

where "L" stands for the leader, to denote the set of all vehicles whose position and velocity vectors are available for the ith agent. We assume that the directed graph of the ensemble of vehicles is such that there exists at least one directed path from the leader to any agent. Note that the leader does not sense any vehicle. Moreover, we assume that 


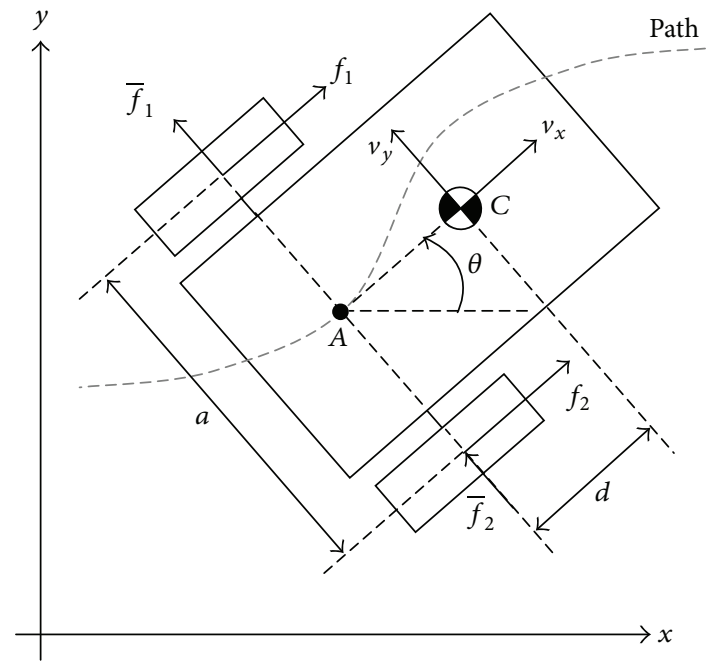

Figure 1: Diagram of a mobile robot.

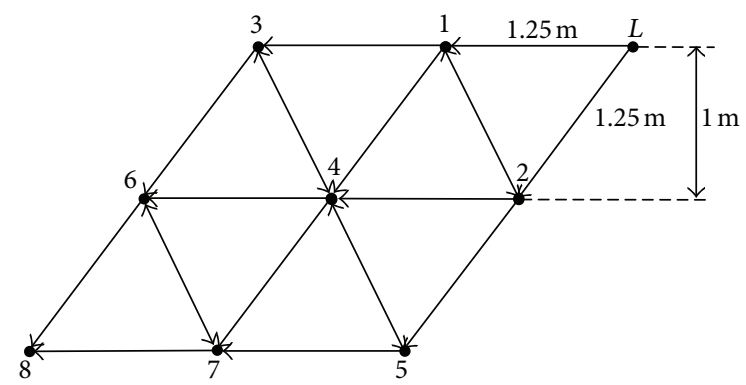

Figure 2: The desired formation of the set of eight robots and a leader with information flow topology.

the graph topology of the vehicle network is time invariant. The normalized directed Laplacian matrix $\mathscr{L} \in \mathbb{R}^{(p+1) \times(p+1)}$ of the graph is defined by (8).

Next, define the position error states as

$$
\begin{aligned}
z_{i}(t) & \triangleq\left[\begin{array}{l}
z_{x i}(t) \\
z_{y i}(t)
\end{array}\right] \\
& =\left[\begin{array}{l}
x_{i}(t)-\frac{1}{\left|\mathscr{F}_{i}\right|} \sum_{j \in \mathscr{F}_{i}}\left(x_{j}(t)+l_{x i j}\right) \\
y_{i}(t)-\frac{1}{\left|\mathscr{F}_{i}\right|} \sum_{j \in \mathscr{F}_{i}}\left(y_{j}(t)+l_{y i j}\right)
\end{array}\right], \quad i=1, \ldots, p, t \geq 0,
\end{aligned}
$$

where $l_{x i j} \in \mathbb{R}$ and $l_{y i j} \in \mathbb{R}$ are the steady state differences between the horizontal and vertical positions, respectively, of the $i$ th and the $j$ th robots. The position error dynamics are obtained by taking the second time derivative of (46) given by

$$
\begin{gathered}
\ddot{z}_{x i}(t)=\widetilde{v}_{1 i}(t)+\mathscr{L}_{i \mathrm{~L}} \ddot{x}_{\mathrm{L}}(t)+\widetilde{g}_{1 i}\left(\widetilde{z}(t), \eta_{2}(t), q_{\mathrm{L}}(t), \dot{q}_{\mathrm{L}}(t)\right), \\
z_{x i}(0)=z_{0 x i}, \quad \dot{z}_{x i}(0)=\dot{z}_{0 x i}, \quad i=1, \ldots, p, t \geq 0,
\end{gathered}
$$

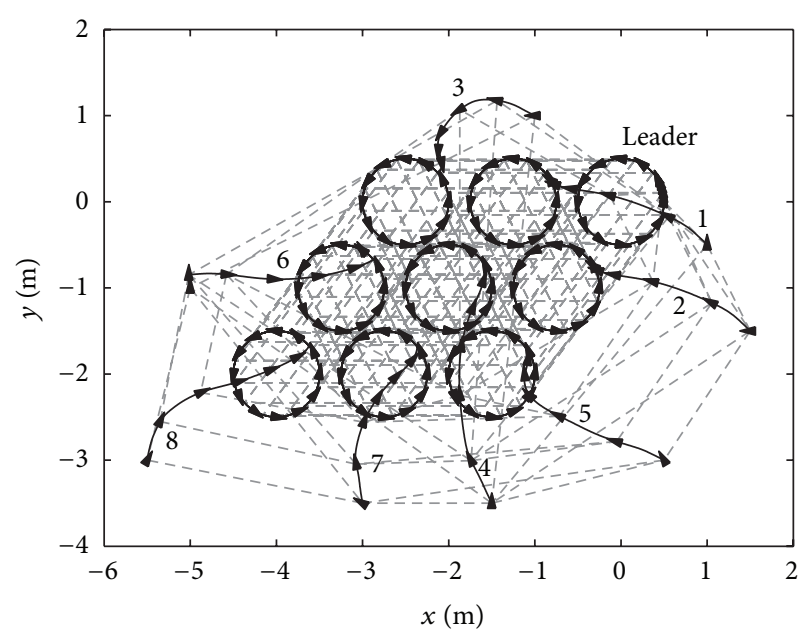

FIgURE 3: Position phase portrait with orientations of eight robots and a leader.

$$
\begin{gathered}
\ddot{z}_{y i}(t)=\widetilde{v}_{2 i}(t)+\mathscr{L}_{i \mathrm{~L}} \ddot{y}_{\mathrm{L}}(t)+\widetilde{g}_{2 i}\left(\tilde{z}(t), \eta_{2}(t), q_{\mathrm{L}}(t), \dot{q}_{\mathrm{L}}(t)\right), \\
z_{y i}(0)=z_{0 y i}, \quad \dot{z}_{y i}(0)=\dot{z}_{0 y i}, \quad i=1, \ldots, p
\end{gathered}
$$

where

$$
\begin{gathered}
\widetilde{z}(t) \triangleq\left[z_{x 1}(t), z_{y 1}(t), \theta_{1}(t), \ldots, z_{x p}(t), z_{y p}(t), \theta_{p}(t)\right]^{\mathrm{T}}, \\
\eta_{2}(t) \triangleq\left[\eta_{21}(t), \ldots, \eta_{2 p}(t)\right]^{\mathrm{T}}, \\
\widetilde{v}_{1 i}(t) \triangleq v_{1 i}(t)-\frac{1}{\left|\mathscr{F}_{i}\right|} \sum_{j \in \mathscr{F}_{i} \backslash\{\mathrm{L}\}}\left(v_{1 j}(t)\right), \\
\widetilde{v}_{2 i}(t) \triangleq v_{2 i}(t)-\frac{1}{\left|\mathscr{F}_{i}\right|} \sum_{j \in \mathscr{F}_{i} \backslash\{\mathrm{L}\}}\left(v_{2 j}(t)\right) .
\end{gathered}
$$

Note that, for $t \geq 0$, leader's position and orientation satisfy

$$
\begin{gathered}
\dot{x}_{\mathrm{L}}(t)=v_{x \mathrm{~L}}(t) \cos \theta_{\mathrm{L}}(t)-d \omega_{\mathrm{L}}(t) \sin \theta_{\mathrm{L}}(t), \\
\dot{y}_{\mathrm{L}}(t)=v_{x \mathrm{~L}}(t) \sin \theta_{\mathrm{L}}(t)+d \omega_{\mathrm{L}}(t) \cos \theta_{\mathrm{L}}(t), \\
\omega_{\mathrm{L}}(t)=-\frac{1}{d}\left(\dot{x}_{\mathrm{L}}(t) \sin \theta_{\mathrm{L}}(t)-\dot{y}_{\mathrm{L}}(t) \cos \theta_{\mathrm{L}}(t)\right),
\end{gathered}
$$

where $\omega_{\mathrm{L}}(t) \triangleq \dot{\theta}_{\mathrm{L}}(t), t \geq 0$. Since, the dynamics of $(47)$ conform with the structure of the error dynamics (13), it follows from Theorem 9 that the sliding mode control (34) guarantees that the solutions to (47) converge to the origin in finite time, and hence all robots reach the desired formation on the plane in finite time. After all robots reached the desired formation at the settling time $T_{s}$, it follows from Lemma 7 that $x_{i}(t)-x_{j}(t)-l_{x i j}=0, y_{i}(t)-y_{j}(t)-l_{y i j}=$ $0, t \geq T_{s}, i, j=\mathrm{L}, 1, \ldots, p$, which implies that $\dot{x}_{i}(t)=$ $\dot{x}_{j}(t), \dot{y}_{i}(t)=\dot{y}_{j}(t), t \geq T_{s}, i, j=\mathrm{L}, 1, \ldots, p$. Thus, the 

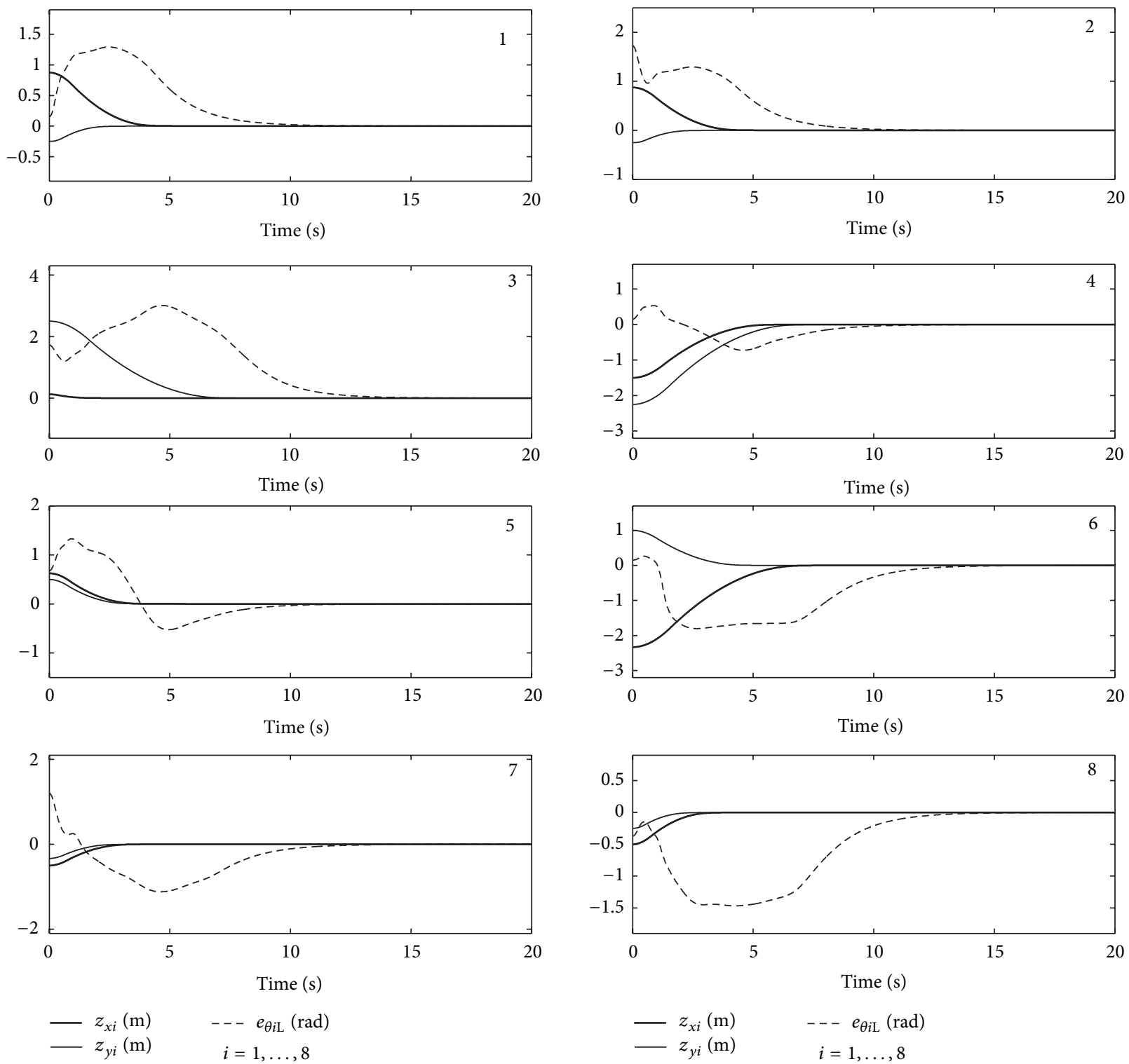

Figure 4: Time history of the errors versus time.

orientation dynamics (37) for the $i$ th robot can be rewritten as

$$
\begin{gathered}
\dot{\theta}_{i}(t)=-\frac{1}{d}\left(\dot{x}_{j}(t) \sin \theta_{i}(t)-\dot{y}_{j}(t) \cos \theta_{i}(t)\right), \\
\theta_{i}\left(T_{s}\right)=\theta_{s i}, \quad t \geq T_{s}, i=1, \ldots, p, j=\mathrm{L}, 1, \ldots, p .
\end{gathered}
$$

Let $e_{\theta i \mathrm{~L}}(t) \triangleq \theta_{i}(t)-\theta_{\mathrm{L}}(t), t \geq T_{s}, i=1, \ldots, p$, measure the orientation error between the $i$ th robot and the leader. Using (50) with $j=\mathrm{L}$, the orientation error dynamics are given by

$$
\begin{gathered}
\dot{e}_{\theta i \mathrm{~L}}(t)=-\frac{1}{d}\left[\dot{x}_{\mathrm{L}}(t) \sin \left(e_{\theta i \mathrm{~L}}(t)+\theta_{\mathrm{L}}(t)\right)\right. \\
\left.-\dot{y}_{\mathrm{L}}(t) \cos \left(e_{\theta i \mathrm{~L}}(t)+\theta_{\mathrm{L}}(t)\right)\right]-\omega_{\mathrm{L}}(t), \\
e_{\theta i \mathrm{~L}}\left(T_{s}\right)=e_{s \theta i \mathrm{~L}}, \quad t \geq T_{s}, \quad i=1, \ldots, p .
\end{gathered}
$$

Alternatively, (51) can be rewritten as

$$
\begin{aligned}
\dot{e}_{\theta i \mathrm{~L}}(t)= & -\frac{1}{d} \dot{x}_{\mathrm{L}}(t)\left(\sin e_{\theta i \mathrm{~L}}(t) \cos \theta_{\mathrm{L}}(t)\right. \\
& \left.\quad+\cos e_{\theta i \mathrm{~L}}(t) \sin \theta_{\mathrm{L}}(t)\right)+\frac{1}{d} \dot{y}_{\mathrm{L}}(t) \\
& \times\left(\cos e_{\theta i \mathrm{~L}}(t) \cos \theta_{\mathrm{L}}(t)-\sin e_{\theta i \mathrm{~L}}(t) \sin \theta_{\mathrm{L}}(t)\right) \\
& \left.-\omega_{\mathrm{L}}(t) \quad+\dot{y}_{\mathrm{L}}(t) \sin \theta_{\mathrm{L}}(t)\right)+\frac{1}{d} \cos e_{\theta i \mathrm{~L}}(t) \\
= & -\frac{1}{d} \sin e_{\theta i \mathrm{~L}}(t)\left(\dot{x}_{\mathrm{L}}(t) \cos \theta_{\mathrm{L}}(t)\right. \\
& \times\left(-\dot{x}_{\mathrm{L}}(t) \sin \theta_{\mathrm{L}}(t)+\dot{y}_{\mathrm{L}}(t) \cos \theta_{\mathrm{L}}(t)\right)-\omega_{\mathrm{L}}(t) \\
= & -\frac{1}{d} v_{x \mathrm{~L}}(t) \sin e_{\theta i \mathrm{~L}}(t)+\omega_{\mathrm{L}}(t)\left(\cos e_{\theta i \mathrm{~L}}(t)-1\right)
\end{aligned}
$$



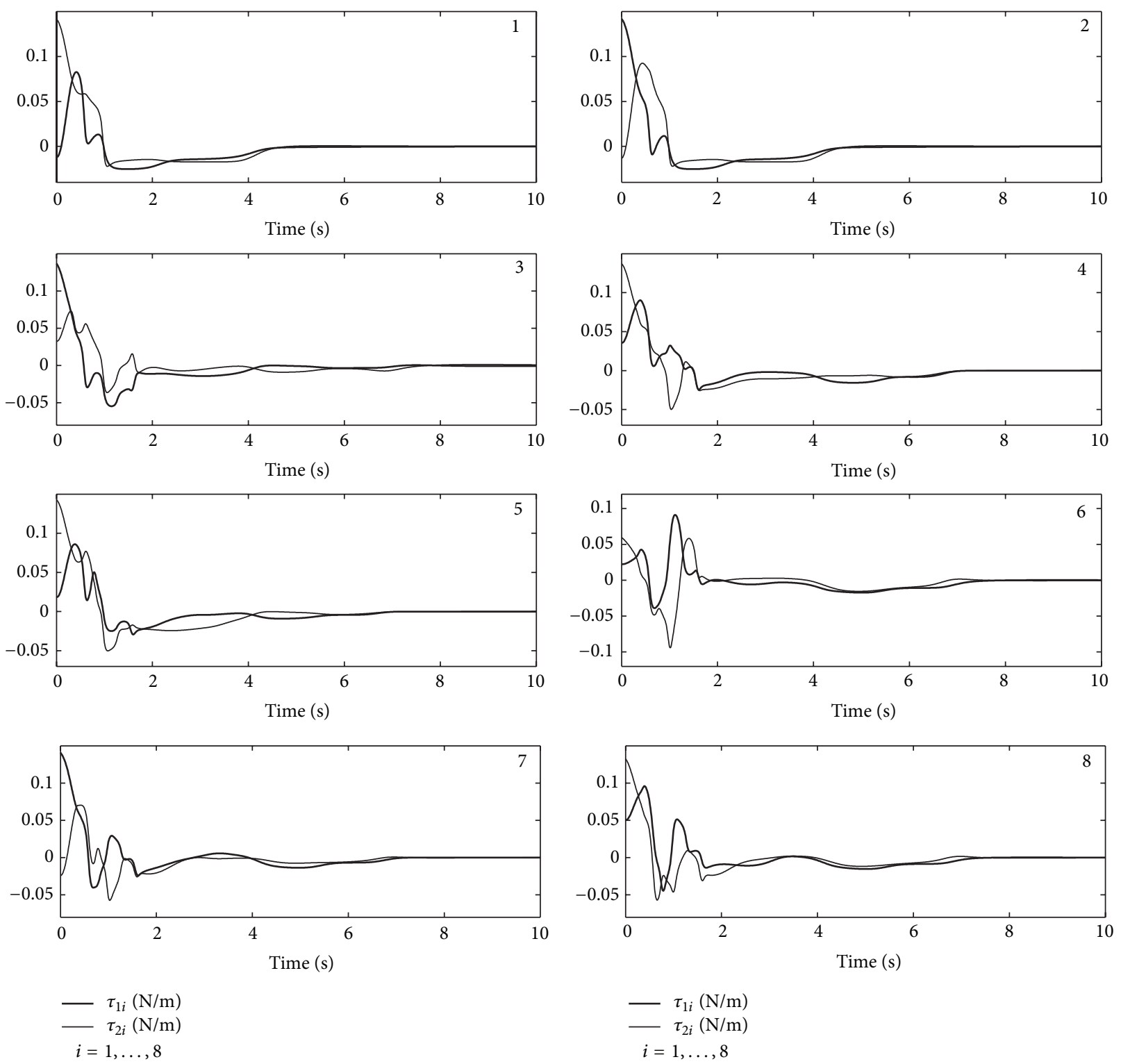

Figure 5: Control torques versus time.

$$
\begin{array}{r}
=-\frac{2}{d} v_{x \mathrm{~L}}(t) \sin \left(\frac{e_{\theta i \mathrm{~L}}(t)}{2}\right) \cos \left(\frac{e_{\theta i \mathrm{~L}}(t)}{2}\right) \\
-2 \omega_{\mathrm{L}}(t) \sin ^{2}\left(\frac{e_{\theta i \mathrm{~L}}(t)}{2}\right) \\
=-\frac{2}{d} \sin \left(\frac{e_{\theta i \mathrm{~L}}(t)}{2}\right)\left[v_{x \mathrm{~L}}(t) \cos \left(\frac{e_{\theta i \mathrm{~L}}(t)}{2}\right)\right. \\
\left.+d \omega_{\mathrm{L}}(t) \sin \left(\frac{e_{\theta i \mathrm{~L}}(t)}{2}\right)\right]
\end{array}
$$$$
=-\frac{1}{d} v_{x \mathrm{~L}}(t) \sin e_{\theta i \mathrm{~L}}(t)\left[1+\frac{d \omega_{\mathrm{L}}(t)}{v_{x \mathrm{~L}}(t)} \tan \left(\frac{e_{\theta i \mathrm{~L}}(t)}{2}\right)\right] \text {, }
$$

$$
e_{\theta i \mathrm{~L}}\left(T_{s}\right)=e_{s \theta i \mathrm{~L}}, t \geq T_{s}, i=1, \ldots, p .
$$

In the next proposition, we show that the zero equilibrium point of (52) is uniformly asymptotically stable with respect to $e_{\theta i \mathrm{~L}}$.

Proposition 11. Consider the error dynamics given by (52) and define

$$
\begin{aligned}
\mathscr{D}_{i} & \triangleq\left\{e_{\theta i \mathrm{~L}} \in \mathbb{R}:\left|e_{\theta i \mathrm{~L}}\right|<2 \operatorname{atan}\left(\left(1-\delta_{\mathrm{i}}\right) \frac{v_{x \mathrm{~L}}^{*}}{\mathrm{~d} \omega_{\mathrm{L}}^{*}}\right)\right\} \\
& \subset(-\pi, \pi), \quad i=1, \ldots, p,
\end{aligned}
$$

where $v_{x \mathrm{~L}}^{*} \triangleq \inf _{t \geq 0}\left(v_{x \mathrm{~L}}(\mathrm{t})\right)>0, \omega_{\mathrm{L}}^{*} \triangleq \sup _{t \geq 0}\left(\left|\omega_{\mathrm{L}}(t)\right|\right)$, and $\delta_{i}>$ $0, i=1, \ldots, p$, is an arbitrarily small constant. Then, the zero solution of (52) is uniformly asymptotically stable with respect to $e_{\theta i \mathrm{~L}}$ and with an estimate of the domain of attraction given by $\mathscr{D}_{i}$. 


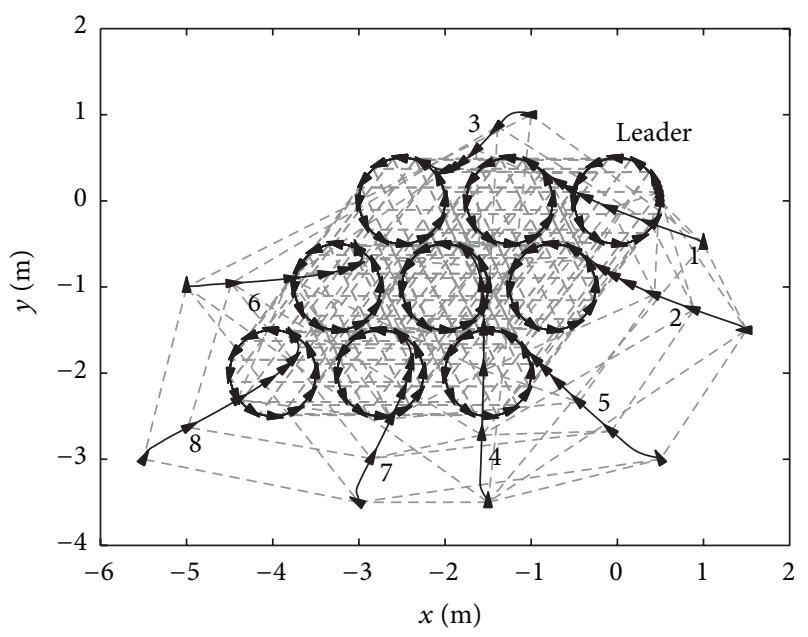

FIGURE 6: Position phase portrait with orientations of eight robots and a leader.

Proof. Note that $\{0\}$ is the only equilibrium point of (52) in $\mathscr{D}_{i}$. Consider a Lyapunov function candidate given by

$$
V_{i}\left(e_{\theta i \mathrm{~L}}\right)=1-\cos e_{\theta i \mathrm{~L}}, \quad e_{\theta i \mathrm{~L}} \in \mathscr{D}_{i}, i=1, \ldots, p,
$$

and note that $V_{i}(0)=0$ and $V_{i}\left(e_{\theta i \mathrm{~L}}\right)>0, e_{\theta i \mathrm{~L}} \in \mathscr{D}_{i}, i=$ $1, \ldots, p$. Furthermore, since $\mathscr{D}_{i} \subset(-\pi, \pi), i=1, \ldots, p$, there exists $\gamma_{i}>0, i=1, \ldots, p$, such that $\left|\sin e_{\theta i \mathrm{~L}}\right|>\gamma_{i}\left|e_{\theta i \mathrm{~L}}\right|, e_{\theta i \mathrm{~L}} \in$ $\mathscr{D}_{i}, i=1, \ldots, p$. Thus, the Lyapunov derivative along the trajectories of (52) satisfies

$$
\begin{aligned}
\dot{V}_{i}\left(e_{\theta i \mathrm{~L}}(t)\right) & =\sin e_{\theta i \mathrm{~L}}(t) \dot{e}_{\theta i \mathrm{~L}}(t) \\
& =-\frac{1}{d} v_{x \mathrm{~L}}(t) \sin ^{2} e_{\theta i \mathrm{~L}}(t)\left[1+\frac{d \omega_{\mathrm{L}}(t)}{v_{x \mathrm{~L}}(t)} \tan \left(\frac{e_{\theta i \mathrm{~L}}(t)}{2}\right)\right] \\
& \leq-\frac{1}{d} v_{x \mathrm{~L}}^{*} \delta_{i} \sin ^{2} e_{\theta i \mathrm{~L}}(t) \\
& \leq-\frac{\gamma_{i}^{2}}{d} v_{x \mathrm{~L}}^{*} \delta_{i} e_{\theta i \mathrm{~L}}^{2}(t), \quad e_{\theta i \mathrm{~L}} \in \mathscr{D}_{i}, i=1, \ldots, p .
\end{aligned}
$$

It follows from Theorem 4.1 of [27] that the zero solution to (52) is uniformly asymptotically stable with respect to $e_{\theta i \mathrm{~L}}$ and with an estimate of the domain of attraction given by $\mathscr{D}_{i}, i=$ $1, \ldots, p$. This implies that $\theta_{i}(t) \rightarrow \theta_{\mathrm{L}}(t)$ as $t \rightarrow \infty$ for all $i=1, \ldots, p$.

Proposition 11 along with Theorem 9 ensures finite-time coordination of the agents' positions and asymptotic coordination of the agents' orientations.

\section{Numerical Example}

In this section, we provide a numerical simulation for the case of planar coordinated motion of eight mobile robots and a leader. The desired formation and the information flow between agents are shown in Figure 2. This communication topology remains unchanged over time. For the given configuration, $L_{1}$ and $L_{2}$ matrices are given as follows:

$$
\begin{aligned}
L_{1} & =\left[\begin{array}{c}
-\frac{1}{2} \\
-\frac{1}{2} \\
0 \\
0 \\
0 \\
0 \\
0 \\
0
\end{array}\right], \\
L_{2} & =\left[\begin{array}{cccccccccc}
1 & -\frac{1}{2} & 0 & 0 & 0 & 0 & 0 & 0 \\
-\frac{1}{2} & 1 & 0 & 0 & 0 & 0 & 0 & 0 \\
-\frac{1}{2} & 0 & 1 & -\frac{1}{2} & 0 & 0 & 0 & 0 \\
-\frac{1}{4} & -\frac{1}{4} & -\frac{1}{4} & 1 & -\frac{1}{4} & 0 & 0 & 0 \\
0 & -\frac{1}{2} & 0 & -\frac{1}{2} & 1 & 0 & 0 & 0 \\
0 & 0 & -\frac{1}{3} & -\frac{1}{3} & 0 & 1 & -\frac{1}{3} & 0 \\
0 & 0 & 0 & -\frac{1}{3} & -\frac{1}{3} & -\frac{1}{3} & 1 & 0 \\
0 & 0 & 0 & 0 & 0 & -\frac{1}{2} & -\frac{1}{2} & 1
\end{array}\right] .
\end{aligned}
$$

Note that the directed graph of the formation satisfies the required property that there exits at least one directed path from the leader to any agent. Also, note that $L_{2}$ is invertible. Next, the leader is set to be moving counterclockwise on a circular path of radius 0.5 according to $x_{\mathrm{L}}(t)=$ $0.5 \cos (\pi t / 30), y_{\mathrm{L}}(t)=0.5 \sin (\pi t / 30), t \geq 0$. The values of control gains are set to be $K_{i}=0.5 \times I_{2}$ and $K_{\text {iaux }}=0.5 \times I_{2}$, $i=1, \ldots, 8$. Finally, the matrices $C_{i} \in \mathbb{R}^{2 \times 2}$ characterizing the design of the sliding surface for each agent are chosen to be $C_{i}=0.5 \times I_{2}, i=1, \ldots, 8$. In this case, $\lambda_{i}=0.75, i=1, \ldots, 8$, ensure that condition (27) is satisfied.

All robots are identical with the values of the parameters set to be $m=2.83 \mathrm{~kg}, I=0.0226 \mathrm{~kg} / \mathrm{m}^{2}, J=5.1 \times 10^{-5} \mathrm{~kg} / \mathrm{m}^{2}$, $a=0.315 \mathrm{~m}, d=0.078 \mathrm{~m}$, and $r=0.045 \mathrm{~m}$. The above values correspond to the experimental wheeled mobile robot parameters which will be discussed in the next section.

For the initial positions and orientations of the robots given by $\eta_{11}(0)=[1,-0.5, \pi / 2]^{\mathrm{T}}, \eta_{12}(0)=[1.5,-1.5,-p i]^{\mathrm{T}}$, $\eta_{13}(0)=[-1,1,-\pi]^{\mathrm{T}}, \eta_{14}(0)=[-1.5,-3.5, \pi / 2]^{\mathrm{T}}$, $\eta_{15}(0)=[0.5,-3,2 \pi / 3]^{\mathrm{T}}, \eta_{16}(0)=[-5,-1, \pi / 2]^{\mathrm{T}}, \eta_{17}(0)=$ $[-3,-3.5,5 \pi / 6]^{\mathrm{T}}$, and $\eta_{18}(0)=[-5.5,-3, \pi / 3]^{\mathrm{T}}$ and the initial velocities given by $\eta_{2 i}=[0,0]^{\mathrm{T}}, i=1, \ldots, 8$, Figure 3 shows the position phase portrait with robot orientations and 

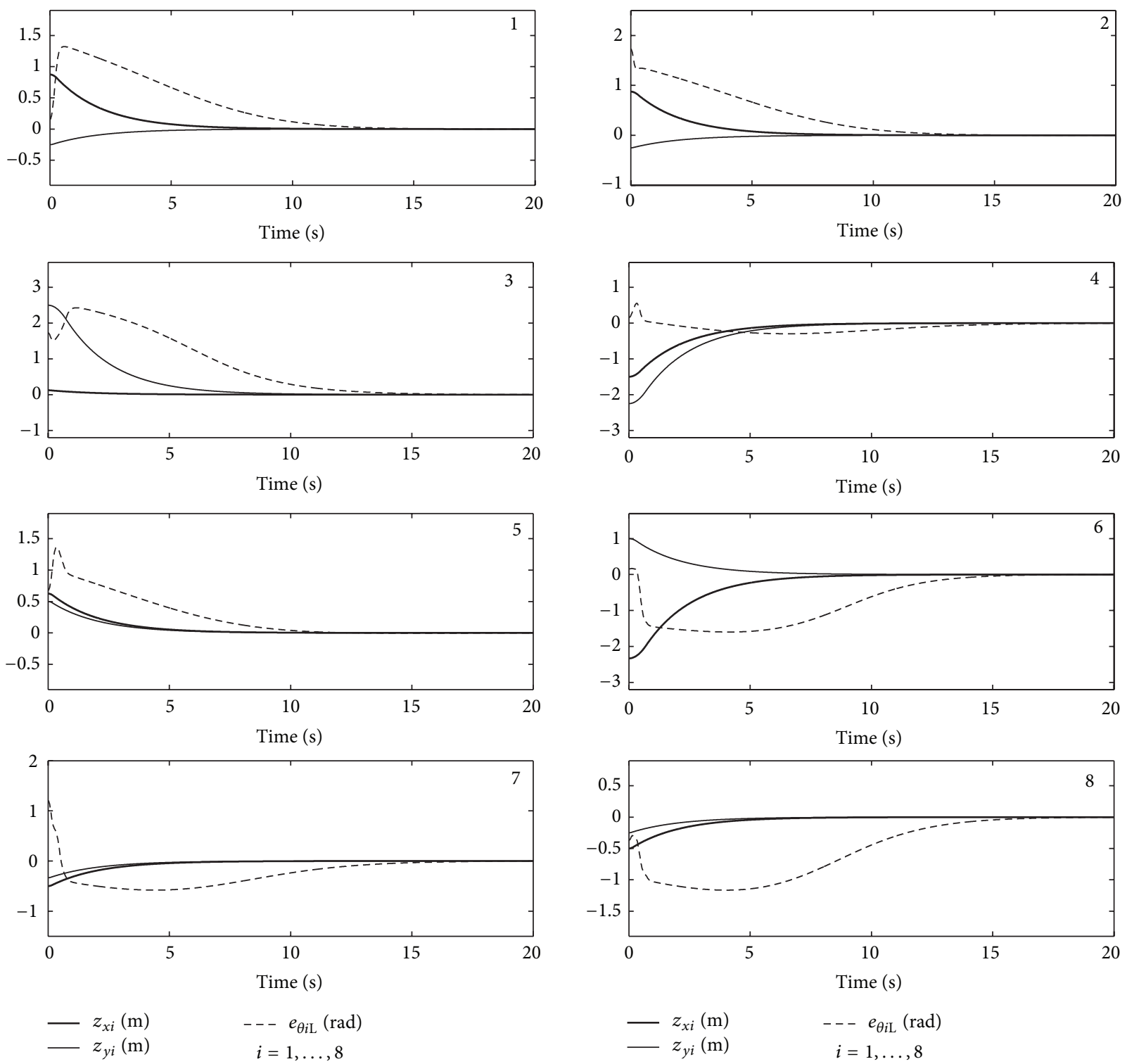

FIgURE 7: Time history of the errors versus time.

Figure 4 shows position errors defined in (46) and orientation errors with respect to the leader for eight robots. It can be seen from Figures 3 and 4 that, with the settling time of approximately 5 seconds, the agents reach the desired formation shown in Figure 2. Furthermore, Figure 4 shows that the errors $z_{x i}$ and $z_{y i}, i=1, \ldots, 8$, converge to zero faster than $e_{\theta i \mathrm{~L}}, i=1, \ldots, 8$, since the former are directly controlled by the feedback control law (34) ensuring finitetime convergence, while the latter is not directly controlled but converges to zero asymptotically. Finally, Figure 5 shows the time history of the control torques acting on both wheels of each robot with $\tau_{1 i}$ and $\tau_{2 i}$ being the control torques acting on the left and the right wheels, respectively, of the $i$ th robot.

Next, we show a comparison between the developed finite-time sliding mode control algorithm and the traditional sliding mode controller predicated on a smooth linear sliding surface. Consider specifically a vector function $\widetilde{\sigma}_{i}: \mathbb{R}^{n} \times$ $\mathbb{R}^{n} \rightarrow \mathbb{R}^{n}$ given by

$$
\widetilde{\sigma}_{i}\left(z_{i}, \dot{z}_{i}\right)=\dot{z}_{i}+\widetilde{C}_{i} z_{i}, \quad\left(z_{i}, \dot{z}_{i}\right) \in \mathbb{R}^{n} \times \mathbb{R}^{n},
$$

where $\widetilde{C}_{i}=\operatorname{diag}\left[\widetilde{c}_{i 1}, \ldots, \widetilde{c}_{i n}\right], \widetilde{c}_{i j}>0, i=1, \ldots, p, j=1, \ldots, n$. We define the $i$ th sliding surface as the null space of $\widetilde{\sigma}_{i}(\cdot, \cdot)$; that is,

$$
\widetilde{\mathcal{S}}_{i} \triangleq\left\{\left(z_{i}, \dot{z}_{i}\right) \in \mathbb{R}^{n} \times \mathbb{R}^{n}: \widetilde{\sigma}_{i}\left(z_{i}, \dot{z}_{i}\right)=0\right\} .
$$

Accordingly, the sliding mode controller can be obtained using the above sliding surface. Next, for this controller, we consider the planar coordinated motion of eight mobile robots and a leader with the same parameter values and the initial conditions as given in the above numerical example. Figure 6 shows the position phase portrait with robot 

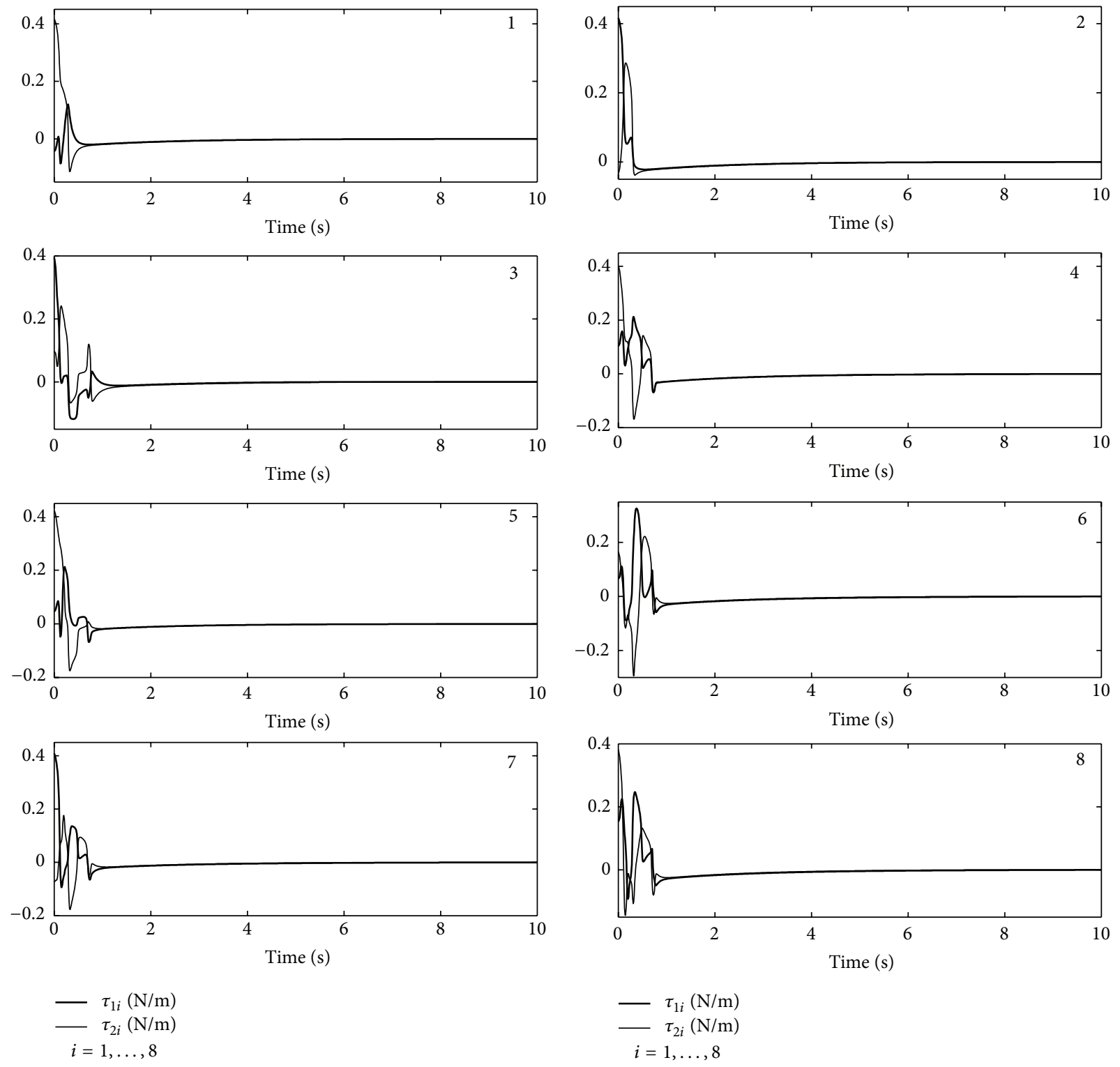

Figure 8: Control torques versus time.

orientations and Figure 7 shows position errors defined in (46) and orientation errors with respect to the leader for eight robots. Finally, Figure 8 shows the time history of the control torques acting on both wheels of each robot with $\tau_{1 i}$ and $\tau_{2 i}$ being the control torques acting on the left and the right wheels, respectively, of the $i$ th robot. The parameters of the sliding surface and the controller are selected such that the settling time for convergence to the desired formation is approximately 5 seconds, that is, the same settling time as for the finite-time sliding mode controller. However, the peak of the control effort for the sliding mode controller predicated on the linear surface (57) is almost three times higher than that of the finite-time sliding mode controller (see Figures 5 and 8) which shows the efficacy of the proposed finite-time control algorithm.

\section{Experimental Validation}

We validate the above results using experimental wheeled mobile robots. The experimental setup consists of an overhead camera, two wheeled mobile robots, and a PC as shown in Figure 9. We use a black and white $640 \times 480$ resolution COHU-2672 camera equipped with a Data Transitions DT3120 frame grabber to capture overhead image frames in order to identify robot position that will be used in our feedback measurements. The camera is installed $2.86 \mathrm{~m}$ above the floor. To ensure that there is no delay throughout the experiment, the camera is set to capture the frames at a sampling rate of $2 \mathrm{fps}$. Since the captured image is distorted, a calibration process is used to allow the true real-world position of every point in the image to be calculated. 


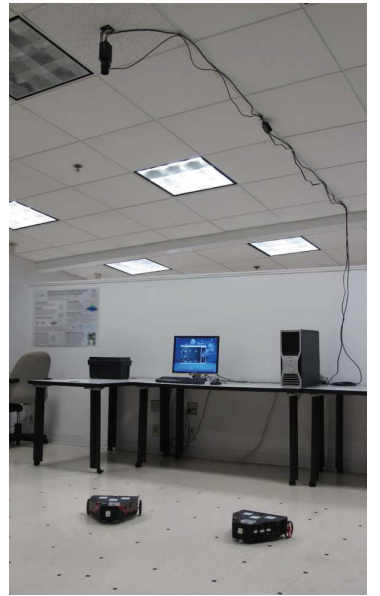

Figure 9: Experimental setup.

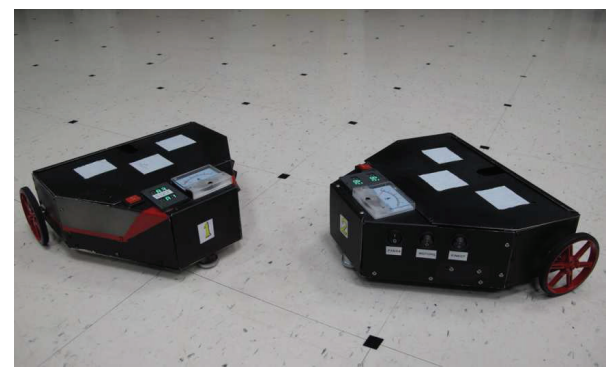

FIGURE 10: Experimental differential drive wheeled mobile robots.

Two wheeled mobile robots used for the experiment are shown in Figure 10. The parameters of these robots are the same as those used in Section 5. In order to detect each robot, three square white labels are attached to its upper surface. Using MATLAB image processing toolbox, the center points of these labels are calculated. The robot orientation is determined by a triangle that these labels form. Moreover, by knowing the robot's center of mass location with respect to any of these labels, the position of the center of mass with respect to the inertial reference frame is obtained by interpolation among the positions of the labels. Finally, the velocity of the robot is determined by taking the time derivative of the filtered position coordinates. The filtering is simply an averaging process since the experiment is implemented at low speed values for the robot.

The robot wheels are driven by two DC motors which are controlled by a Pandaboard single-board computer running a Linux operating system and an Arduino microcontroller board. The control program is built in The MathWorks Simulink environment. Through the Simulink Real-Time Workshop, the program is compiled and built as a real-time executable for the Pandaboard target [28]. The communication between the PC computer and the Pandaboard is done via wireless connection. The communication signals contain computed control velocity commands, specifically, the angular velocities for the wheels. These control velocity commands
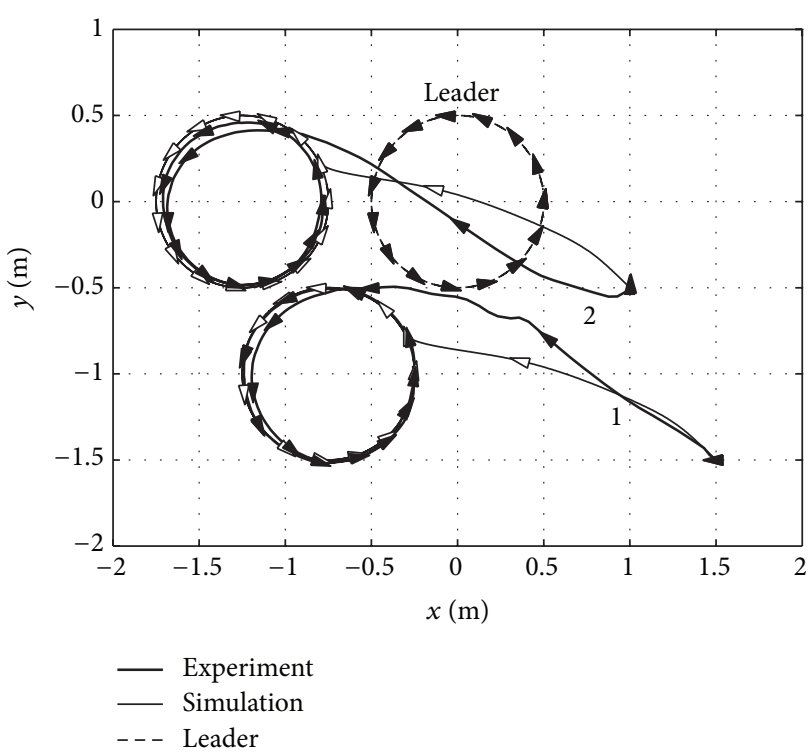

FIGURE 11: Position phase portrait with orientation of the robots.
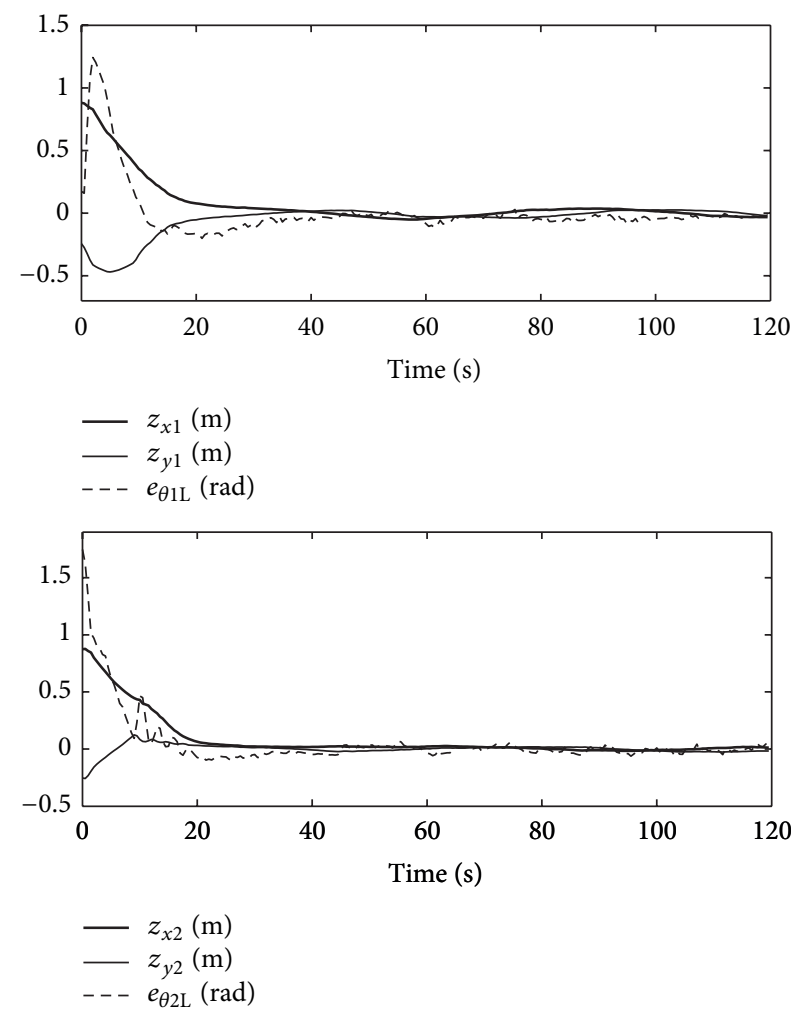

Figure 12: The time history of the errors.

are generated by integrating the differential torques obtained using the coordination control algorithm.

For the following experiment, we consider the coordination problem discussed in Section 5 with the first and the second agents in Figure 2 being our experimental wheeled mobile robots. Figure 11 shows the position phase portrait 


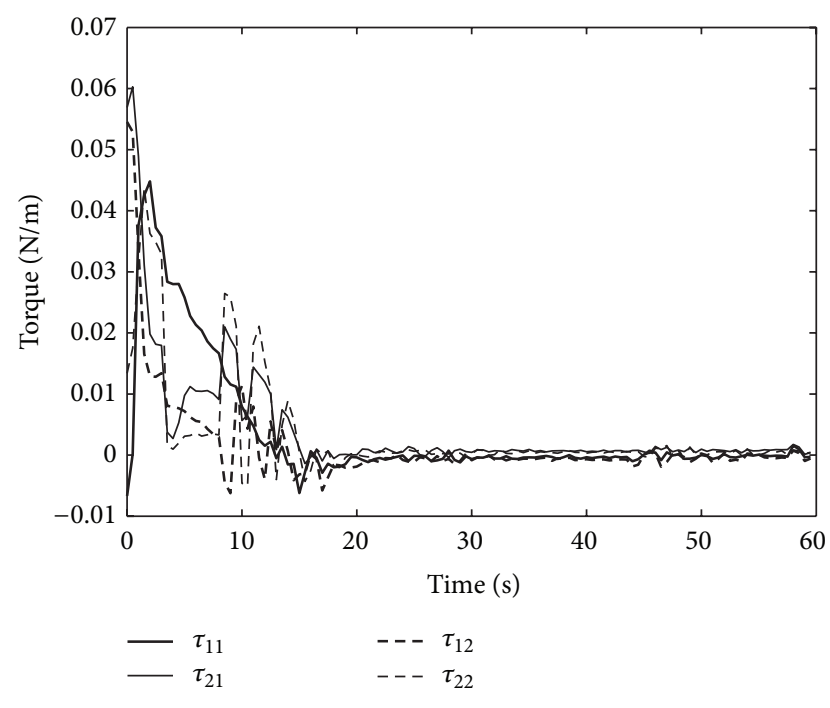

FIGURE 13: Control torques versus time.

with the robots orientations and Figure 12 shows position and orientation errors of the robots. Figure 13 shows the time history of the control torques acting on both wheels of the robot with $\tau_{1}$ and $\tau_{2}$ being the control torques acting on the left and the right wheels, respectively.

In order to suppress chattering induced mostly by the sampling rate of the camera, we used a less sharp (than in the simulations in the previous section) arctangent estimate of the signum function in the controller. Therefore, it is expected that the robots in the experiment converge to the desired formation slower than those in the simulation. As can be seen from Figures 11, 12, and 13, the experimental results match the trajectory profiles from the simulation (Figures 3, 4, and 5), although with a longer convergence time due to the change in the signum function approximation and saturation. This validates the proposed control algorithm even in the presence of implementation issues.

\section{Conclusion}

In this paper, we developed a decentralized sliding mode control technique that guarantees finite-time coordination in multiagent systems. We considered specifically a group of dynamical agents along with a leader with an objective to reach and maintain the desired formation in finite time. Using graph-theoretic notions, we characterized such formation via a directed graph Laplacian matrix and developed individual controllers that use only local information from the current agent and the neighboring agents that directly communicate their information to the current agent. The key feature of our sliding mode control is in the design of nonsmooth sliding surfaces that allow for finite-time convergence of the error states to the origin. The above results were developed for multiagent systems composed of fully actuated EulerLagrange systems and were further extended to address underactuated agent dynamics. A numerical example along with experimental validation was presented to show the efficacy of the approach.

\section{Acknowledgment}

This research was supported in part by the College of Engineering at Villanova University.

\section{References}

[1] S. P. Bhat and D. S. Bernstein, "Finite-time stability of continuous autonomous systems," SIAM Journal on Control and Optimization, vol. 38, no. 3, pp. 751-766, 2000.

[2] S. P. Bhat and D. S. Bernstein, "Geometric homogeneity with applications to finite-time stability," Mathematics of Control, Signals, and Systems, vol. 17, no. 2, pp. 101-127, 2005.

[3] V. T. Haimo, "Finite time controllers," SIAM Journal on Control and Optimization, vol. 24, no. 4, pp. 760-770, 1986.

[4] S. P. Bhat and D. S. Bernstein, "Continuous finite-time stabilization of the translational and rotational double integrators," IEEE Transactions on Automatic Control, vol. 43, no. 5, pp. 678-682, 1998.

[5] Y. Hong, "Finite-time stabilization and stabilizability of a class of controllable systems," Systems \& Control Letters, vol. 46, no. 4, pp. 231-236, 2002.

[6] Y. Hong, J. Huang, and Y. Xu, "On an output feedback finitetime stabilization problem," IEEE Transactions on Automatic Control, vol. 46, no. 2, pp. 305-309, 2001.

[7] S. G. Nersesov, C. Nataraj, and J. M. Avis, "Design of finitetime stabilizing controllers for nonlinear dynamical systems," International Journal of Robust and Nonlinear Control, vol. 19, no. 8, pp. 900-918, 2009.

[8] R. Diestel, Graph Theory, vol. 173 of Graduate Texts in Mathematics, Springer, New York, NY, USA, 1997.

[9] J. A. Fax and R. M. Murray, "Information flow and cooperative control of vehicle formations," IEEE Transactions on Automatic Control, vol. 49, no. 9, pp. 1465-1476, 2004.

[10] W. Ren and Y. Cao, Distributed Coordination of Multi-Agent Networks. Emergent Problems, Models, and Issues, Springer, London, UK, 2011.

[11] W. M. Haddad, V. Chellaboina, and S. G. Nersesov, Thermodynamics. A Dynamical Systems Approach, Princeton Series in Applied Mathematics, Princeton University Press, Princeton, NJ, USA, 2005.

[12] V. I. Utkin, "Variable structure systems with sliding modes," IEEE Transactions on Automatic Control, vol. 22, no. 2, pp. 212222, 1977.

[13] R. A. DeCarlo, S. H. Zak, and G. P. Matthews, "Variable structure control of nonlinear multivariable systems: a tutorial," Proceedings of the IEEE, vol. 76, no. 3, pp. 212-232, 1988.

[14] C. Edwards and S. Spurgeon, Sliding Mode Control: Theory and Applications, Taylor and Francis, London, UK, 1998.

[15] I. Boiko, Discontinuous Control Systems: Frequency-Domain Analysis and Design, Birkhäuser, Boston, Mass, USA, 2009.

[16] Y. V. Orlov, Discontinuous Systems: Lyapunov Analysis and Robust Synthesis under Uncertainty Conditions, Communications and Control Engineering Series, Springer, London, UK, 2009.

[17] Y. Shtessel, C. Edwards, L. Fridman, and A. Levant, Sliding Mode Control and Observation, Control Engineering, Birkhäuser, New York, NY, USA, 2014. 
[18] M. Ghasemi and S. Nersesov, "Finite-time coordination in multiagent systems using sliding mode control," in Proceedings of the American Control Conference, pp. 2053-2058, Washington, DC, USA, June 2013.

[19] R. P. Agarwal and V. Lakshmikantham, Uniqueness and Nonuniqueness Criteria for Ordinary Differential Equations, vol. 6 of Series in Real Analysis, World Scientific Publishing, River Edge, NJ, USA, 1993.

[20] A. F. Filippov, Differential Equations with Discontinuous Righthand Sides, vol. 18 of Mathematics and Its Applications (Soviet Series), Kluwer Academic Publishers, Dordrecht, The Netherlands, 1988.

[21] M. Kawski, "Stabilization of nonlinear systems in the plane," Systems \& Control Letters, vol. 12, no. 2, pp. 169-175, 1989.

[22] T. Yoshizawa, Stability Theory by Liapunov's Second Method, Publications of the Mathematical Society of Japan, no. 9, The Mathematical Society of Japan, Tokyo, Japan, 1966.

[23] F. Bullo, J. Cortés, and S. Martínez, Distributed Control of Robotic Networks, Princeton Series in Applied Mathematics, Princeton University Press, Princeton, NJ, USA, 2009.

[24] M. Mesbahi and M. Egerstedt, Graph Theoretic Methods in Multiagent Networks, Princeton Series in Applied Mathematics, Princeton University Press, Princeton, NJ, USA, 2010.

[25] J. S. Caughman and J. J. P. Veerman, "Kernels of directed graph Laplacians," The Electronic Journal of Combinatorics, vol. 13, no. 1, pp. 1-8, 2006.

[26] S. Nersesov and M. Ghasemi, "Finite-time tracking for EulerLagrange systems using sliding mode control," in Proceedings of the ASME Dynamic Systems and Control Conference, Fort Lauderdale, Fla, USA, October 2012, paper no. DSCC2012MOVIC2012-8665.

[27] W. M. Haddad and V. Chellaboina, Nonlinear Dynamical Systems and Control. A Lyapunov-Based Approach, Princeton University Press, Princeton, NJ, USA, 2008.

[28] J. Fabian and G. Clayton, "One-point visual odometry using a RGB-depth camera pair," in Proceedings of the ASME Dynamic Systems and Control Conference, Fort Lauderdale, Fla, USA, October 2012, paper no. DSCC2012-MOVIC2012-8700. 


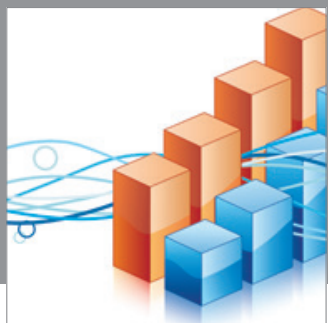

Advances in

Operations Research

mansans

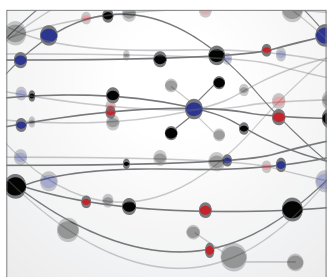

The Scientific World Journal
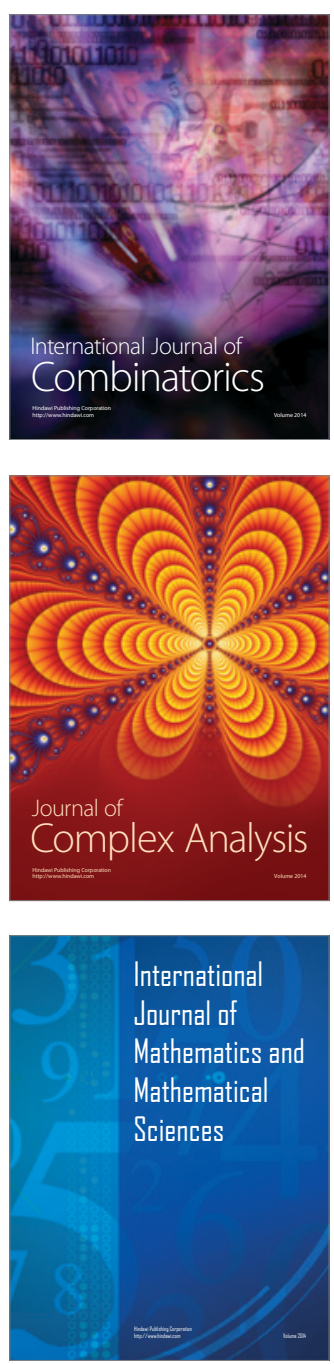
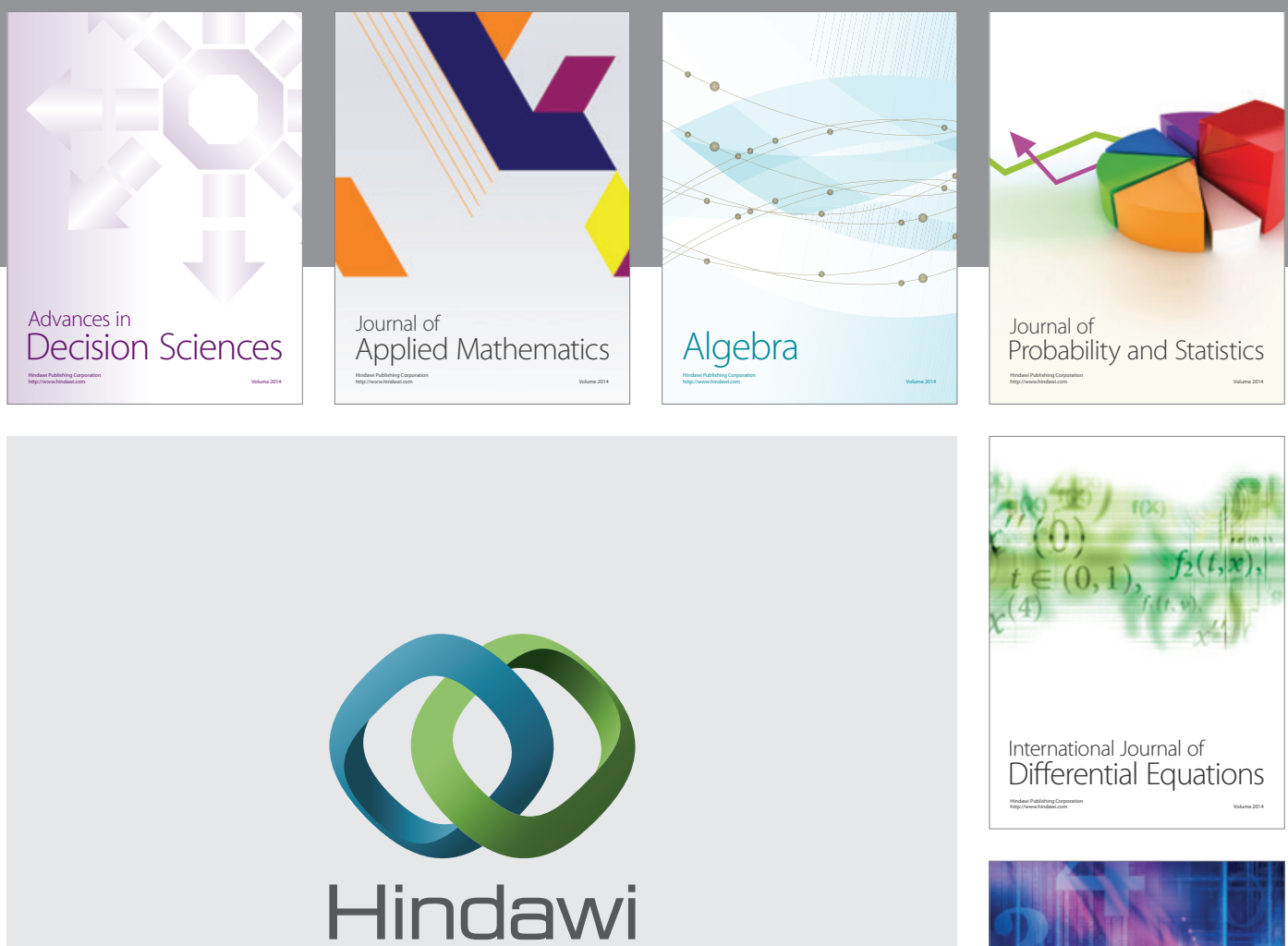

Submit your manuscripts at http://www.hindawi.com
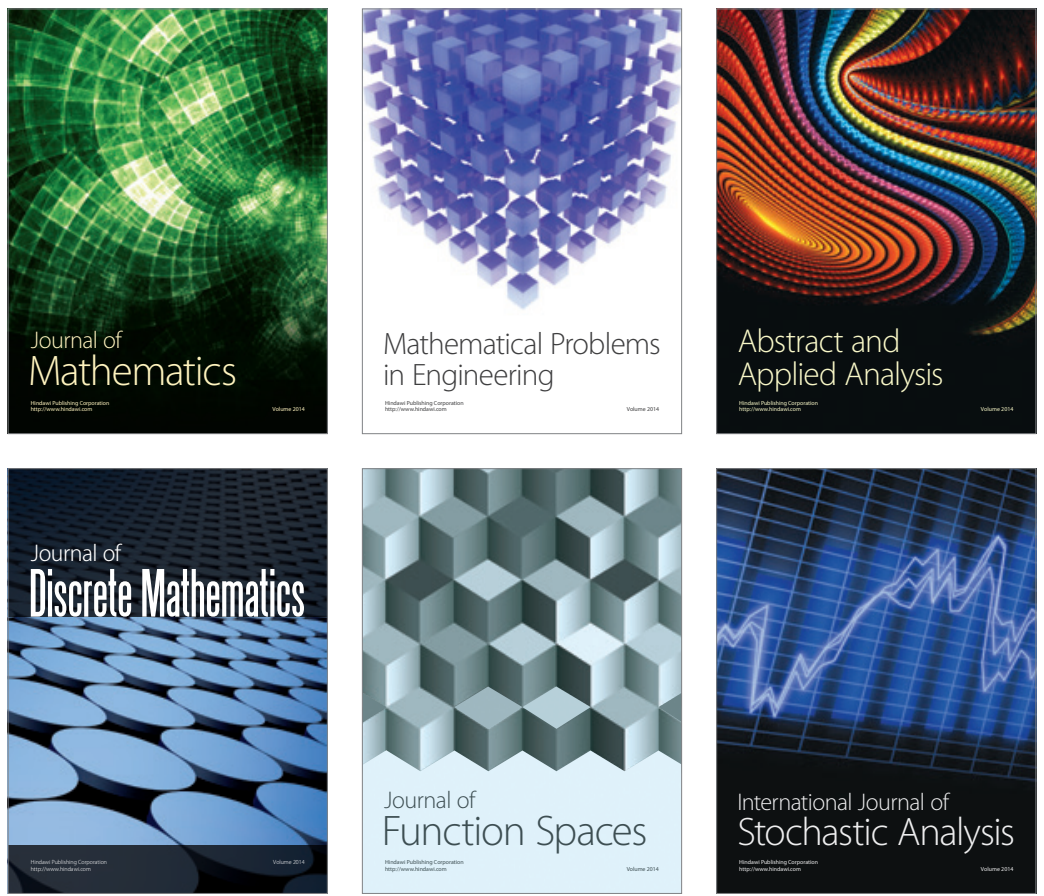

Journal of

Function Spaces

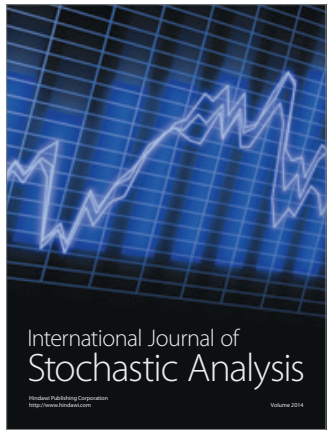

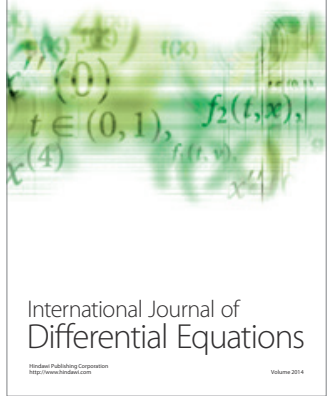
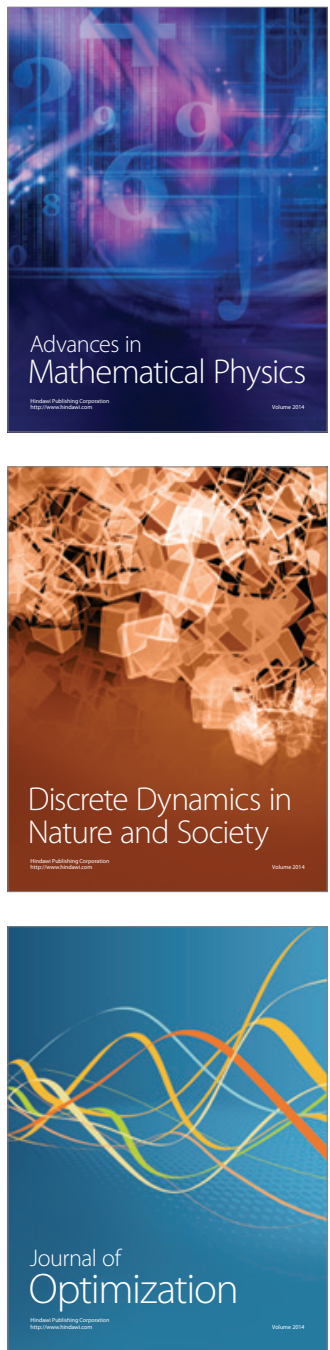